\title{
Kidney failure during HIV disease treated with tenofovir, multiple concurrent diseases and drug therapies
}

Roberto Manfredi ${ }^{1}$, Leonardo Calza ${ }^{1}$, Vincenzo Colangeli ${ }^{1}$, Nicola Dentale ${ }^{1}$, Gabriella Verucchi ${ }^{1}$

1 Department of Internal Medicine, Ageing, and Nephrologic Sciences, Division of Infectious Diseases, "Alma Mater Studiorum" University of Bologna, S. Orsola-Malpighi Hospital, Bologna, Italy

\section{Abstract}

A significant case report of a HIV infected patient in his fifties who experienced an excellent virological and immunological response to antiretroviral therapy (which has been modified just to prevent or avoid some adverse events), but developed a severe, sudden acute kidney failure while under a polypharmacy due to some underlying and overwhelming disorders (i.e. arterial hypertension, non-insulin-dependent diabetes mellitus, a recent acute heart infarction with remarkable remnants, and finally an anecdotal muscle-joint pain with self-prescription of non-steroideal anti-inflammatory drugs), represents the key point for a debate around the increasing frequency of "polypharmacy" in the field of HIV infection, even when HIV resistance to antiretroviral is not a concern. The continuing increase of mean age of HIV-infected population, plus the existing, sometimes unmodifiable risk factors for cardiovascular, dysmetabolic, and renal disorders, plus the adjunct of anecdotal illnesses prompting the resort to different drugs and medications, either prescribed for HIV infection itself, or taken for concurrent or subsequent diseases, or self-prescribed occasionally due to an intercurrent, trivial disorders per se, may prompt a complicated scenario culminating with a lifethreatening acute renal failure of tubular origin. Our report gives us the opportunity to revise and discuss the expected interactions between antiretroviral therapy and the even growing exposure to multiple different drug and drug classes, which may be responsible for relevant drug interactions and direct or adjunctive end-organ impairment, up to life-threatening conditions, which may be avoided or prevented by considering carefully all comorbidites and co-treatments potentially administered to HIV infected patients, thirty years after the discovery of AIDS.

\section{Keywords}

HIV infection; Antiretroviral treatment; Tenofovir; Acute renal failure; Comorbidities; Toxicity; Drug surveillance 


\section{Background}

Even though the introduction of the combined antiretroviral therapies (cART) significantly contributed to a rapid and huge drop of the overall morbidity and mortality rates of HIV disease since around 15 years ago (year 1996, when the first "triple therapies" containing the HIV protease inhibitors became available), however we are experiencing an increasing burden of a very broad spectrum of organ and tissue damages and/or dysfunctions, often severe and sometimes life-threatening in their clinical expression, which appeared to be related to many factors, which are often not independent, one with another: HIV infection itself, HIV-related disorders, drugs administered to treat HIV and other administered drugs, increased mean age, life-style factors and patient's genetic background.

The present review aims at discussing the influence of all these factors. The starting point is a clinical case report observed by the authors, which gives us the opportunity to revise and discuss the expected interactions between antiretroviral therapy and the even growing exposure to multiple different drug and drug classes, which may be responsible for relevant drug interactions and direct or adjunctive endorgan impairment, up to life-threatening conditions, which may be avoided or prevented by considering carefully all comorbidities and co-treatments potentially administered to HIV infected patients, thirty years after the discovery of AIDS.

Some points of discussion emerging from the occurred case report are addressed and commented in the following literature review and discussion, which focuses on extremely significant pharmacological and nephrological-internal medicine problems encountered in the daily management of HIV disease, from a general and broad point of view.

\section{Case report and clinical question}

A 51-year-old male patient with a familiar history of cardiovascular disease (but a negligible personal and familiar history with regard to diabetes mellitus and nephropathy), who has been smoking around 20 cigarettes per day since over 30 years, had a history of moderate alcohol intake at main meals (without prior or present illicit drug abuse), a body mass index and a waist-to-hip abdominal circumference within normal limits, and a mild arterial hypertension treated successfully since around three years with the fixed association valsartan $(160 \mathrm{mg})$ plus hydrochlorotiazide $(12.5 \mathrm{mg})$, was incidentally diagnosed with an heterosexually-transmitted HIV infection six years and eight months ago, at our dedicated outpatient centre. At that time, he felt well, and no relevant clinical problems were present, save another sexually-transmitted disease, i.e. a latent syphilis, which was immediately and successfully treated with i.m. benzylpenicillin, according to standard indications.

Soon (12 weeks) after the diagnosis of HIV infection (when his baseline HIV-RNA level was 84,000 copies $/ \mathrm{ml}$, and his absolute T-lymphocyte count proved to be $322 \mathrm{cells} / \mu \mathrm{l}-23 \%$ of the total T-lymphocyte count), a cART regimen was started according with present international guidelines, with associated zidovudine-lamivudine (as a fixed combination), plus the fixed protease inhibitor association lopinavir-ritonavir (as the standard boosted HIV protease inhibitor, at that time - year 2003). Virological success (as expressed by the achievement of undetectable viral load levels, set at < 200 HIV-RNA copies/ml at that time) was attained three months later, while the immunological recovery allowed our patient to reach his peak of absolute peripheral $\mathrm{CD} 4^{+} \mathrm{T}$-lymphocyte count $(626 \mathrm{cells} / \mu \mathrm{l} ; 26 \%$ of absolute T-lymphocytes), compared with the baseline value of 322 cells/ $\mu \mathrm{l}(23 \%), 12$ months after starting the first-line cART regimen.

Subsequently, during a quite long (46-month) period, two therapeutic switches of cART were deemed necessary, due to antiretroviral drug(s) intolerance, prevention of expected toxicities, enhancement of patient's convenience, and consequently achievement of the best possible adherence. 
Neither virologic nor immunological nor clinical failures emerged during this entire period, and no HIV-associated disorders of any kind were present or were detected, since ever.

No prominent toxicities developed during the first cART regimen (zidovudine-lamivudine, plus lopinavir-ritonavir), when excluding a mild hypercholesterolemia (maximum serum levels of cholesterol $220 \mathrm{mg} / \mathrm{dl}$, with HDL cholesterol at $44 \mathrm{mg} / \mathrm{dl}$, and LDL cholesterol $175 \mathrm{mg} / \mathrm{dl}$ ), mostly prompted by lopinavir-ritonavir, and treated successfully with rosuvastatin (at only $5 \mathrm{mg} /$ day), and a more evident, lopinavir-ritonavir-linked hypertriglyceridemia (up to $292 \mathrm{mg} / \mathrm{dl}$ of serum triglycerides reached six months after the introduction of this fixed-dose HIV protease inhibitor combination), successfully managed with diet, physical exercise, and polyunsaturated fatty acids supplementation (at $4 \mathrm{~g}$ per day), only, until values around $200 \mathrm{mg} / \mathrm{dl}$ of serum triglyceride levels were steadily maintained. At that time, we avoided the administration of fibrates and other hypolipidemic medications registered for the pharmacological treatment of hypertriglyceridemia, since they are burdened by potentially serious drugdrug interactions with cART and other concomitant medicines, especially statins.

When coming to the last 22 months, our patient has developed some remarkable and worsening clinical problems, when treated with his fifth cART line.

In fact, the second proposed regimen, which was a combination of efavirenz plus the fixed nucleoside association zidovudine-lamivudine, was not tolerated, due to persistent central nervous system (CNS) subjective symptoms, and a hitchy but self-limited maculo-papular cutaneous rash, probably related to efavirenz administration, while the nucleos(t)ide backbone, and the other concurrent medications remained unchanged.

At that time, in accordance with novel drug availabilies and the updated antiretroviral therapy guidelines, the staff physician of our dedicated HIV outpatient centre recommended the fixed nucleos(t)ide association of two nucleos(t)ide reverse transcriptase inhibitors (tenofovir-emtricitabine, 245-200 mg once daily), and the protease inhibitor saquinavir (1000 mg twice daily), plus ritonavir booster (100 mg twice daily).

Looking for a cART regimen simplification and a reduction of ritonavir booster dosage (which was reponsiblle for a moderate diarrhea and nausea-vomiting), the previous cART regimen was switched after nine months in order to introduce another protease inhibitor-based cART (i.e., atazanavir $300 \mathrm{mg}$ once daily, plus a "baby"-mimimum dose of ritonavir = $100 \mathrm{mg}$ /day), to support the already effective patient's adherence, to overcome the patient's "intolerance" to ritonavir, and to reduce the pill burden, too.

Nine months later, a further protease inhibitor-based regimen (i.e., fosamprenavir $700 \mathrm{mg}$ twice daily, plus ritonavir booster $100 \mathrm{mg}$ twice daily) was deemed necessary, after which our patient developed an otherwise asymptomatic, but persisting jaundice (which is an expected and characteristic adverse event of atazanavir, whose clearance occurs after hepatic glucuroconjugation). Under the atazanavir-based regimen, the patient's serum bilirubin levels rose up to 7,66 $\mathrm{mg} / \mathrm{dl}$, and did not decrease significantly over three consecutive standard quarterly clinical-laboratory controls made at our outpatient centre.

A slight peripheral (facial and limb) HIV- and cART-related lipoatrophy was present since a couple of years, as better depicted by a Dual-Energy X-ray Absorptiometry (DEXA) scan, as mean of excluding relevant alterations of bone mineral density, and of ruling out an evident visceral lipoaccumulation.

A severe, sudden thoracic pain occurred when our patient was driving his car, and going to making his job.

It was immediately diagnosed at an emergency unit of another Hospital of Bologna, Italy, as an acute myocardial infarction, which required a prompt coronary artery angioplasty with stent placement in the left anterior descending coronary artery, and concurrent thrombolytic treatment and clopidogrel administration (subsequently followed by the introduction of aspirin at the standard $100 \mathrm{mg} /$ day dose). Notwithstanding the rapid and effective cardiologic management, the myocardial infarction resulted in a seriously impaired left ventricular systolic function, as expressed by an ejection fraction steadily reduced to around $30 \%$. 
During the subsequent clinical and laboratory follow-up, three months later a mild fasting serum glucose level elevation $(121 \mathrm{mg} / \mathrm{dl})$ prompted the timely measurement of HbAlc $(7.2 \%)$, so that a diagnosis of frank diabetes mellitus was also posed, on the basis of the laboratory control plasma insulin, C-peptide, and fructosamine levels. The diagnosis of diabetes mellitus was enforced after detecting significant alterations at the standard oral glucose load tolerance testing (OGTT). As a result, a type II non-insulin-dependent diabetes mellitus was defined, and a specific diet plus physical exercise program (shared with cardiology consultants), and the oral antidiabetic agent metformin (at $850 \mathrm{mg}$, two times a day) were prescribed.

Unfortunately, a concurrent (hypertension-related? diabetes mellitus-related? HIV-related? tenofovirrelated? multiple drug-related? accelerated atherogenesis-related?), apparently slight "nephropathy" was disclosed for the first time, based on a protein-creatinine ratio of 1.2, whereas the kidney function appeared still fully preserved, as assessed on the ground of trivial serum creatinine levels ranging from 0.8 to $1.1 \mathrm{mg} / \mathrm{dl}$ in two laboratory controls obtained in one week, and creatinine clearance values varying from 85 to $99 \mathrm{ml} / \mathrm{min}$, while serum and urine electrolytes, serum osmolarity, and all the other parameters of urinalysis, remained within standard limits for the entire observation period.

Beyond the well established and well tolerated antiretroviral therapy steadily performed with tenofovir/ emtricitabine plus fosamprenavir/ritonavir, which ensured a persistingly stable virological suppression (viral load always $<50$ HIV-RNA copies/ml), and a perfectly maintained immune system recovery (as expressed by a $\mathrm{CD} 4^{+}$cell count persistingly beyond 550 cells $/ \mu$, with the $\mathrm{CD} 4+$ lymphocyte rate always over 27\%), after the mentioned examinations and the novel diagnoses, multiple medications were added, upon specialistic consultation with cardiologists, diabetologists, and nephrologists, which were promptly ensured during the first week following the patient's recovery from the acute heart infarction, and his subsequent hospital discharge.

These medications were: metformin ( $850 \mathrm{mg}$ twice daily) for the recently diagnosed non-insulin-dependent diabetes mellitus type II, plus aspirin (100 mg/day), carvedilol (12.5 mg twice daily), enalapril ( $2.5 \mathrm{mg}$ twice daily), and furosemide (at $25 \mathrm{mg}$ once daily), for the underlying, and very recent heart ischemic injury with notable post-infarction remnants, the appearance of very mild (almost negligible) disorders of kidney function, and the already known arterial hypertension, which always remained perfectly under control (with the former valsartan/hidrochlorotiazide therapy interrupted, when a betablocker, plus an ACE-inhibitor, plus a mild dose of the most common renal loop diuretic furosemide were added), upon triple cardiologic-diabetologic-nephrologic specialistic consultancies were obtained. During the next five months, our patient remained substantially stable, save the need of a mild increase of metformin dosage (prescribed at $500 \mathrm{mg}$ thrice daily, at main meals), in order to keep serum HbA1c always within its normal threshold, associated two months later with a doubled furosemide dosage ( $25 \mathrm{mg}$ twice daily), in order to control a mild edema of lower limbs, probably related to the patient's prolonged standing, when at work.

At that time, a repeated heart ultrasonography showed for the first time an impairment of the right ventricular function, while the ejection fraction of the left ventricle remained substantially stable (around $30 \%)$.

Suddenly and unexpectedly, 13 days after his previous scheduled consultation at our outpatient centre and his antiretroviral drug refill and careful check of all concurrent drug prescriptions and medications, our patient after calling us by telephone, came directly to the Hospital, asking for an urgent visit. $\mathrm{He}$ complained of deep asthenia, generalized malaise, polypnea and nausea without fever, vomiting, and other thoracic and gastrointestinal signs and symptoms, lasting and progressing since three days. After telling (among others), that he strained his back during the past week when at work, he declared the spontaneous assumption of over-the-counter ibuprofen (already present at patient's home), taken at a dosage varying from 400 to $800 \mathrm{mg} /$ day for five consecutive days. Notwithstanding a complete rest recommended by the primary care physician, and his self-medication with an over-the-counter 
NSAID, he has felt progressively worse and worse, until the above-mentioned request of an urgent outpatient visit.

Our patient was initially visited by the physician in charge at our HIV outpatient centre, and showed a substantially negative physical examination, when excluding a frank polypnea (25-30 breaths per minute) and a clearly accelerated arterial pulse rate (120 per minute), but hemoglobin $\mathrm{O}_{2}$ saturation tested 99\% without oxygen therapy, and neither increased body temperature, nor an abnormal arterial blood pressure, were present. The electrocardiography assay tested comparable with previous controls (i.e. a prevalence of left ventricle potentials over right ones, a probable mild right atrium enlargement, plus aspecific repolarization alterations, in absence of any ischemic sign).

In a few minutes, we rapidly moved to our Day-Hospital rooms.

At this facility of ours, it was possible to make an urgent, standard chest X-ray examination, and an abdominal ultrasound assessment (which did not show significant abnormalities, when excluding a mild enlargement of the heart frame, without any alteration of great vessels of lung parenchyma, and pleura, and especially intrabdominal organs, with special attention deserved to kidneys and urinary tract), together with an urgent blood and blood gas examination, which was technically executable at our Day-Hospital also after the scheduled early morning time established by our Hospital reference laboratory for the standard outpatient subject controls (i.e. 9:00 am).

Early in the afternoon, the urgent laboratory values of our patients were as follows: serum glucose 133 $\mathrm{mg} / \mathrm{dl}$, serum creatinine $1.19 \mathrm{mg} / \mathrm{dl}$, blood urea nitrogen $81 \mathrm{mg} / \mathrm{dl}$, sodium $139 \mathrm{mEq} / \mathrm{l}$, potassium 6.5 $\mathrm{mEq} / \mathrm{l}$ (while all other available hematological and biochemistry analyses, including serum troponin, myoglobin, and creatinphosphokinase levels, proved perfectly normal). The patient's urgent urinalysis did not show albumin, urinary tract cells, red and white blood cells, and bacteria, fungi, or crystals, the urine $\mathrm{pH}$ was 7.5, but specific gravity tested slightly low: i.e. 1.009. An arterial blood gas examination made concurrently with the other urgent laboratory tests, due to persisting dyspnea and hyperventilation, without any very significant clinical and radiological clue, detected: normal $\mathrm{pO}_{2}$ and $\mathrm{pCO}_{2}$ values, while bicarbonates proved $14.1 \mathrm{~g} / \mathrm{dl}$ (i.e. features suggestive of metabolic acidosis). Serum lactates were not required, while further laboratory examinations (including i.e. the potentially relevant serum/urine phosphate rate, other serum/urine electrolytes, and serum osmolarity) could not be measured immediately, since they are not included in the panel of "urgent" basic laboratory examinations which may be performed "as default" tests in non-hospitalized patients, and answered in 90-120 minutes time maximum. Immediately after preliminary laboratory examinations became available on the internal web network of our University Hospital (S. Orsola-Malpighi, Bologna, Italy), the patient was hospitalized at our inpatient Division in the early evening of the same day, for further examinations and treatment, as appropriate.

During the next few hours, when all pharmacological treatments had been temporarily withdrawn, the diagnosis of metabolic acidosis of suspected "renal" origin was confirmed. Elevated serum lactate levels $(41 \mathrm{mg} / \mathrm{dl})$ were disclosed by standard examinations, with a relevant hypokaliemia (serum potassium $2.9 \mathrm{mmol} / \mathrm{l}$ ) with a concurrent, mild hypophosphoremia, and slightly reduced serum calcium levels, while urinalysis showed a prominent increase of potassium, phosphorus, and calcium concentration, with a lowered output of sodium and chloride.

During the first 24-36 hours of hospitalization, serum kidney enzymes (i.e. serum creatinin, urea, and uric acid levels), moderately worsened, but they were rapidly corrected by a prompt supportive care (progressive fluid refill, and bicarbonate and electrolyte administration titred every 2-6 hours on the ground of clinical and laboratory testing and urinalysis), while all vital parameters and the hourly urine output remained within normal limits. A complete resolution was attained in the subsequent 72 hours (at the sixth day since admission), which was followed by the expected a 5-7-day-long hypostenuric polyuria which reflected the temporarily impaired tubule function due to the sudden, extensive renal tubule necrosis occurred one week before. After 12 comprehensive days of hospitalization, our patient 
was discharged without any kind of renal function sequelae, which did not appear during the subsequent, 16-month follow-up, until now.

In early March, 2011, our patient is still stable on his novel cART association including: the fixed dose tenofovir/emtricitabine (at one pill, once daily), re-introduced one week after discharge, and the protease inhibitor darunavir (at $800 \mathrm{mg}$ once daily), plus ritonavir (at $100 \mathrm{mg} /$ daily), which replaced the previous protease inhibitor association of fosamprenavir plus ritonavir. In particular, tenofovir (as well as all other concurrent medications) had been withdrawn upon hospital admission, but it was safely re-introduced only one week after discharge without any other clinical and laboratory problem in the subsequent 16-month follow-up, while acting as a potent, safe, and convenient once-daily therapy in its fixed-dose backbone combination with emtricitabine. Concurrently, the protease inhibitor darunavir, recently approved as a first-line choice also in patients without previous virological failures, was successfully introduced without any significant adverse event of any kind, as checked for the same 16-month observation period. The fixed "backbone" association of tenofovir/emtricitabine was therefore maintained (depending on a careful and strict patient's monitoring in the first weeks), since its use was weighted against the potential risks of the other available fixed associations of nucleos(t)ide analogue backbones, i.e. that of abacavir plus lamivudine, and that of zidovudine and lamivudine. When selecting a "third" agent, in order to "restore" a "classical" triple cART combination in a patient who achieved a complete and sustained virological response to all previous cART combination thanks to his $100 \%$ adherence to all regimens, never experienced a virological and/or immunological and/or clinical failure in his entire life, and was invited or "forced" to switch even five times his previous "classical" cART regimens due to mild and/or transient adverse events, and/or aiming to further improve the patient's convenience and to reduce the global pill burden, a rechallenge with another non-nucleoside reverse transcriptase inhibitor was avoided (due to previous patient's intolerance to efavirenz), so that the last-generation once-daily protease inhibitor darunavir was selected, since it perfectly conjugates an elevated potency, a high genetic barrier to HIV mutation and resistance, and enhanced dysmetabolic, end-organ, and also renal safety profile, especially when given as a single daily dose of $800 \mathrm{mg}$ only, plus the less possible ritonavir booster dosage (100 mg/day). In early March 2011 our patient is still on his last tenofovir-emtricitabine plus darunavir-ritonavir cART, he maintains a steadily suppressed HIV viremia (plasma HIV-RNA levels $<50$ copies/ml), and an absolute CD4+ lymphocyte count of 602 cells/ $\mu$ l concurs (i.e. $28 \%$ of absolute peripheral T-lymphocytes), at our last available clinical and laboratory control of February 27, 2011.

With regard to the multiple, concomitant medications which played a life-saving role in our patient (all major non-HIV-related disorders!), since over 16 months ago we confirmed rosuvastatin (at $5 \mathrm{mg}$ / day) to maintain serum total cholesterol levels and cholesterol fractions under the enforced thresholds for patients who already experienced a major cardiovascular event, plus omega-3 polyunsaturated fatty acids (always at $4 \mathrm{~g} /$ day), from the dysmetabolic point of view, and as a part of a mandatory secondary cardiovascular prevention subsequent to a the major accident (the prior acute heart infarction). When considering the stable left heart ventricle impairment established in our patient, a treatment with the ACE-inhibitor ramipril ( $5 \mathrm{mg}$ /day), the beta-blocker metoprolole (at $100 \mathrm{mg}$ daily), plus low-dosage furosemide $(25 \mathrm{mg} /$ day), and aspirin $(100 \mathrm{mg} /$ day $)$ was successfully continued without any clinical and/or laboratory disturbance, together while low-dose pioglitazone (15 mg/day only), which was selected instead of metformin as an oral antidiabetic drug for the underlying non-insulin-dependent diabetes mellitus, which remained perfectly under control from a clinical, laboratory, and instrumental point of view (with regard to serum glucose levels, Hblc threshold, urinalysis, and ophthalmologic and neurological examinations carried our by our consultants). Over the entire observation period which followed the discharge from our inpatient service (over 16 months ago), the patient's kidney function remained perfectly stable and within normal limits, and microalbuminuria and other diabetes- and hypertension-related disorders never appeared in our quarterly laboratory and clinical controls. 


\section{Causes of kidney failure in HIV patients}

Causes can be summarized as follows:

- HIV infection itself, including directly- and indirectly-mediated organ and tissue damage [1-11];

- HIV-related disorders, which showed a dramatic decline of their incidence and overall progression and mortality rates just during the cART era, thanks to the effective antiviral activity of cART, and the immune recovery prompted by the same highly active anti-HIV treatments. However, an increasingly modified disease presentation, which may lead to a true "pathomorphism" of infectious, neoplastic, and other HIV-related and AIDS-related and unrelated disorders in an increasing number of cases, has been recognized just since the cART era. As known, this relevant phenomenon has emerged in parallel with the use of effective and potent cART regimens, and it may be attributable to a very extensive number and variety of concurrent, and not always independent factors and co-factors [8,12-20], including immune recovery achieved just thanks to cART itself $[21,22]$, and worldwide epidemiological changes (due to huge immigration flows, for example) [23]. At the same time, the number of "AIDS presenters", i.e. the subjects in whom HIV disease has been detected concurrently with one or more AIDS-defining disorders, is increasing worldwide, and has become an extremely worrying clinical and especially public health concern, in its dimensions and implications [12-15,18,24-27];

- the role of administered drugs, and especially that determined by cART itself, and their multiple, varied, and often associated organ and tissue toxicities, which add to pre-existing HIV- and nonHIV-correlated disorders and their respective pharmacological treatments [1-5,8,10,18-20,28-47]. The so-called "lipodistrophy syndrome" is one of the key features also heralding a significantly greater risk of cardiovascular and other end-organ events, including vascular structure and function, bone and mineral metabolism, with kidney function obviously interested [20,48-51];

- other medicines of any kind, prescribed with an increasing frequency over years and especially during the cART era, in an increasing proportion of patients, also to prevent or manage the extensive toxicities of cART itself and that of other, concomitant medications [3,6,7,8,10,18,28-33,39,4446,52-63] (Table I);

- lifestyle-related relevant population-based [64,65], or individually recognized factors, including i.e. cigarette smoking, illicit or recreational drugs use, alcohol intake, lack of an appropriate diet and physical exercise, i.e. a broad series of "unmodifiable" and "modifiable" risk factors for cardiocerebrovascular damage and other potentially severe end-organ disorders among HIV-infected patients, as known since many years [8,10,61,66-68];

- the progressively increased mean age of the entire population living with HIV, which unavoidably predisposes to further comorbidities and to an exponential increase of nested disorders, as well as the expected cumbersome problems linked to their prevention, monitoring, and management in the daily clinical practice of physicians engaged in the care of people affected by HIV disease, even more during the third millennium $[4,5,7,8,10,18,19-22,27,28,34-36,41-43,61,69,70]$;

- the genetic background of patients, taken as racial- and gender-related issues, and individual features, which may influence and affect all the above-mentioned causes and correlations $[10,18,21,22,70-75]$.

\section{ART regimens and renal function}

With regard to renal function and kidney disorders, all approved cART regimens according to the present, updated 2010-2011 guidelines, regardless of their composition, significantly reduce the overall 
mortality and deaths related to all severe kidney diseases in patients with HIV infection. However, the proportion of chronic and end-stage renal disease seems on the rise over time just from the early cART era - year 1996) $[6,7,18-20,43,47,62,76]$. For instance, the so-called "HOPS cohort" study which included nearly 7,000 HIV-infected patients followed per a median time of over 39 months, showed that the proportion of deaths involving kidney disorders significantly increased from the year 1996 to 2004 [7]. Consequently, the cumulative risk of developing either acute or chronic renal injuries, and subsequently end-stage renal disease in a non-negligible proportion of patients, remains proportionally elevated. Moreover, these kidney disorders are probably missed or diagnosed late and underreported (especially when mild events are of concern), and remain largely underestimated in current clinical practice. In addition, cART itself and the role of underlying diseases and that of concomitant medications, may contribute to bias this figure in any possible direction. Anyway, early, borderline, subtle, or near-negligible abnormalities of renal function parameters and serum-urine electrolyte levels may be detected frequently during the overall natural history of HIV disease, even more among patients receiving cART regimens, and further more when other diseases and other medications, or lifestyle or elderly themselves, are of some concern [5,6-8,10,18,19,21,22,28,36,42,43,46,54,61-63,76], so that it becomes exceedingly difficult to distinguish between "para-physiological" conditions prompted by occasional circumstances (i.e. a trivial dehydration occurring during a hot Summer, and the paradoxical immune recovery prompted by cART itself) [21,22], and subtle abnormalities which may precipitate into a fullblown renal or end-organ disorder, which may sometimes prompt a life-threatening event $[63,77,78]$.

\section{Tenofovir}

Tenofovir disoproxil fumarate (briefly, tenofovir), has been discovered through a collaborative research project between Antonín Holý at the Institute of Organic Chemistry and Biochemistry, Academy of Sciences of Prague (Czech Republic), and Erik DeClercq, of the Rega Institute for Medical Research, at the Catholic University of Leuven, Belgium. Tenofovir has been approved by the United States Food and Drug Administration (FDA) on October 26, 2001, for the treatment of HIV infection [10,79], and nearly 7 years later (on August 11, 2008), for the treatment of chronic hepatitis B [80,81]. Tenofovir is a first-choice nucleoside reverse transcriptase inhibitor for the treatment of HIV infection in the large majority of current therapeutic lines, because of its intrinsic antiviral potency, its safety profile, its convenient once-daily dosage (also as fixed combination with emtricitabine, and even more as a triple fixed association including the first-choice non-nucleoside reverse transcriptase inhibitor efavirenz), and its elevated potency against $\mathrm{HBV}$ infection too, which is a frequent comorbidity just relevant for patients living with HIV infection [10,43,76,80-82].

The "intrinsic" tenofovir-associated nephrotoxicity has been extensively and quite well studied $[10,18,43,47,62,74-76,79,83,84]$. Like other nucleos(t)ide analogues, tenofovir requires intracellular phosphorilation to become pharmacologically active, and at the cell level several different drug carriers may act on the intracellular concentration and disposition of all these drugs [74]. In particular, tenofovir transportation occurs through proximal tubular cells, by the action of four so-called organic anion transporters (OATs), whose types 1 and 3 are mostly involved for drug uptake. The renal elimination of tenofovir is provided by a cluster of 14 multi-drug resistance-associated proteins (MRPs), the most relevant of which are MRP-2 (also called ABCC2), and MRP-4 (also named ABCC4) [74]. Notably, just MRP-2 activity is blunted by the HIV protease inhibitor ritonavir (regardless of its dosage, i.e. $100 \mathrm{mg} /$ day as atazanavir or darunavir booster, or $400 \mathrm{mg}$ /day as tipranavir booster). Furthermore, several genetic polymorphisms of these transporters may affect their function, with unpredictable consequences on intracellular tenofovir concentrations, and its directly related toxic effects [74,75].

The kidney toxicity of tenofovir usually involves the proximal tubule function, but other mechanisms prompted by the frequent underlying comorbidities and related polypharmacology, and the increasing life expectancy of subjects living with HIV may reveal, unmask, increase, complicate, and finally lead 
to an acute kidney failure, or to a progressive renal damage evolving into an end-stage kidney disease, which either could not be prevented, or had been previously neglected, or has not been discovered earlier, due to an endless number of possible causes and interferences [5,8,10,18,19,36-45,57-63,85-87]. When associated with the other nucleos(t)ide analogue emtricitabine (in the mentioned fixed combination), or with lamivudine as a first-line nucleos(t)ide analogue backbone component of a "classical" cART regimen [82], largely employed also in HIV-HBV-co-infected patients, and also when administered to HBV-mono-infected patients $[81,82,89]$, tenofovir results safe in the large majority of cases. This concept remains true when tenofovir is used alone, as well as when it is prescribed in combination with HIV non-nucleoside reverse transcriptase inhibitors (like efavirenz, nevirapine, and etravirine) [88], and novel-class anti-HIV agents like integrase inhibitors (i.e. raltegravir and elvitegravir), entry inhibitors (like maraviroc and vicriviroc), and fusion inhibitors (like enfuvirtide). On the other hand, some nucleos $(\mathrm{t})$ ide analogues (like didanosine, whose plasmatic levels increase upon co-administation wih tenofovir) $[10,38]$, and also the antiviral ribavirin for the treatment of chronic hepatitis $\mathrm{C}$ [69,89-92], the "older", intrinsically nephrotoxic protease inhibitor indinavir $[44,45,93,94]$, as well as the presently used first-line protease inhibitor-based cART regimens (especially those including ritonavir booster), might add subtle, but sometimes significantly kidney toxicity concerns [10,18-20,3739,43-45,60,62,71,76,85,86,88,93,94]. The "intrinsic" (pharmacologically determined), but somewhat negligible and reversible tenofovir nephrotoxicity, could be also enhanced by concomitant disorders, and even more by many concurrent medications chronically or acutely prescribed (or spontaneously taken by patients themselves), for their known, underlying chronic disorders (either associated with HIV disease and cART itself, or not), their age-related disorders, or their occasional, mild-to-moderate intercurrent or incidental illnesses, even trivial in relevance (but treated with either prescription medications or self-prescribed, over-the-counter drugs, like the non-steroideal anti-inflammatory drugs - NSAIDs) [10,18,19,43,60,76,93].

\section{HIV acute nephropathy}

The most severe HIV-associated nephropathy (the so-called HIV acute nephropathy, or "HIVAN"), is found in the majority of cases among Africans and African descents, usually shortly after acquiring HIV infection, which has been hypothesized to act directly or indirectly on this vital organ [3,6$9,63,95]$. HIVAN shows a rapid progression to end-stage renal disease when antiretroviral therapy is not available, or is not given promptly. As expected, the administration of cART is known to reverse the natural history of HIVAN, but the kidney benefits of cART may not be limited to HIVAN only. Unfortunately, we are aware that cART is often underprescribed or incorrectly dosed or taken non only in developing countries, just where HIVAN is more frequent $[63,95]$, but also in industrialized countries $[96,97]$, even more in persons with chronic kidney disorders, with or without a concurrent HIV disease $[10,18,19,83,95,98]$.

As anticipated, the direct effects of HIV infection on the kidney sum up with a varied genetic background and an extremely broad spectrum of immune-mediated factors, physiological conditions like pregnancy [75], and especially underlying comorbidities, immune recovery due to cART itself $[10,21,22,63]$, and especially overwhelming (also non-HIV associated) diseases, the frequent chronic co-infections (i.e. chronic hepatitis B, D, and especially C) [90-92], but also an increasing prevalence of sexually-transmitted diseases including syphilis [24,99-101] (as in our case), and even hepatitis A [102].

As expected, the extremely different medications prescribed (or self-prescribed, or taken in a not appropriate, even "heterodox" mode by HIV-infected patients themselves) $[28,83,96-98,103]$ have their intrinsic toxicities but they also have potential, varied drug-drug interactions among an almost endless list of drugs potentially used (or useful, or needed, or taken as "recreational" ones) by individuals living with HIV, even more in the years 2010-2011, when the life expectancy of HIV-infected individuals is approaching that of the general population (Table I) $[5,8,18,27,36,42,67]$. 


\begin{tabular}{|c|c|c|c|}
\hline Drugs & $\begin{array}{l}\text { Acute tubular injury } \\
\text { (ATI) or AKI }\end{array}$ & $\begin{array}{l}\text { Acute interstitial } \\
\text { nephritis }\end{array}$ & $\begin{array}{l}\text { Other associated } \\
\text { abnormalities }\end{array}$ \\
\hline TMP-SMX (Bactrim $\left.{ }^{\circledR}\right)$ & & +++ & Hyperkalemia, crystalluria \\
\hline$\beta$-lactams & & ++ & \\
\hline Sulfadiazine & & ++ & Crystalluria, nephrolithiasis \\
\hline Fluoroquinolones & & + & \\
\hline Rifampin & + & + & Hypokalemia, hypouricemia, hypernatremia, vasculitis \\
\hline $\mathrm{INH}$ & & + & Overdose leads to high anion gap metabolic acidosis \\
\hline NSAIDs & $+/-$ & + & $\begin{array}{l}\text { Proteinuria, secondary minimal change disease, } \\
\text { papillary necrosis }\end{array}$ \\
\hline Dapsone & $+/-$ & & Papillary necrosis \\
\hline Amphotericin B & +++ & & Hypokalemia, hypomagnesemia, hypernatremia, NDI \\
\hline Pentamidine & +++ & & Crystalluria, hyperkalemia \\
\hline Foscarnet & +++ & & Hypercalcemia/hypernatremia, Glomerular crystals \\
\hline Ganciclovir & + & & \\
\hline Acyclovir & + & $+/-$ & Crystalluria \\
\hline Indinavir, atazanavir & & + & Crystalluria, nephrolithiasis \\
\hline Abacavir & & $+/-$ & \\
\hline Tenofovir & ++ & & Fanconi, NDI \\
\hline
\end{tabular}

Table I. Selective drugs causing AKI in HIV-infected patients [104]

$+=$ mild injury; $++=$ moderate injury; $+++=$ severe injury; INH = isoniazid; NDI = nephrogenic diabetes

insipidus; $\mathrm{SMX}=$ sulfamethoxazide; $\mathrm{TMP}=$ trimethoprim

This explosive "polypharmacy" typical of a growing rate of patients living with HIV disease, may prompt a proportionally enlarged spectrum of end-organ damages, also including serious kidney disorders, too [5-7,10,18,19,36-43,54,57-63,66,69,76,85,86], just at a greater extent among HIV-infected individuals, who already suffer from their expected problems of prompt recognition, expert diagnosis, careful clinical and laboratory management, strict monitoring, and possibly "proactive" prevention measures of all toxicities and adverse events, which could be avoided or blunt as far as possible $[8,10,18,19,62]$.

In our specific case report, and in our specific situation, we have to review some key points of interest for either specialists of different medical specialties, and practitioners (family care physicians):

\section{Familiar background}

Some familiar background for cardiovascular diseases as a whole (which is very common in the general population of North-Eastern Italy - Emilia-Romagna region, whose capital city is Bologna) [64,65], some relevant lifestyle habits (i.e. cigarette smoking since many years, moderate alcohol consumption at major meals), but still normal body mass index and waist-to-hip circumference, an already established essential arterial hypertension successfully treated, followed by a sudden, overwhelming major heart event such as an acute myocardial infarction with important end evolving sequelae, plus the "incidental discovery" of a type II, non-insulin-dependent frank diabetes mellitus, deserving oral antidiabetics only, is the "evolving clinical picture" of our unfortunate patient;

\section{Lifetime pharmacological treatments}

The requirement of multiple, chronic (quoad vitam, i.e. lifetime) pharmacological treatments, including common anti-hypertensive drug combinations (which had been switched from the former ba- 
seline valsartan/hidrochlorotiazide association towards a beta-blocker plus an ACE-inhibitor, introduced after the acute myocardial infarction and successfully maintained after the acute episode of the described kidney insufficiency), the mandatory adjunct of $100 \mathrm{mg}$ aspirin after the acute myocardial infarction with relevant sequelae, and the continued, very simple loop diuretics (like furosemide in our case, whose initial dosage had been initially increased due to a mild, concurrent edema of lower limbs of non-nephrogenic nature, and then continued also after the acute kidney failure at lower dosage), as well as oral antidiabetic agents (with the intercurrent resort to an increased dosage of metformin, in order to maintain our patient within the desirable Hblc threshold just before his "critical" episodes of acute renal failure).

The oral antidiabetic therapy was subsequently changed towards the more recent oral thiazolidinedione insuline-sensitizing agent pioglitazone, administered at its lower dosage of $15 \mathrm{mg} /$ day. This oral antidiabetic agent has been selected with careful attention after and instead of previous metformin therapy, since it was thought to add something to recover (and maybe prevent?) the HIV- and cART related peripheral lipoatrophy (which is part of the very common lipodistrophy syndrome of many patients living with HIV, but was present in a very mild form in our patient) [49,105-107]. Since according to a recent meta-analysis, the "cousin" thiazolidinedione rosiglitazon, which showed a very significant activity in recovering just tenofovir nephrotoxicity [91], but has not demonstrated significantly greater beneficial effects on the lipodistrophy syndrome over both pioglitazone and especially metformin [106], pioglitazone seems safer in patients burdened by a high cardio-cerebrovascular risk (like our patient), although the previously employed metformin proved the only insulin-sensitizer agent which has been demonstrated to partly improve visceral fat accumulation, serum lipid profile, and also endothelial function in the general population (but no data are available until now in HIV-infected patients). Anyway, we decided to favor pioglitazone (and not rosiglitazone), in our patient, who received it at the same dosage for over 16 consecutive months obtaining a full control of his diabetes mellitus, in absence of significant adverse events (including the already present mild limb edema, which remained unchanged despite pioglitazone therapy, which has lower limb edema listed among its potential side effects). A substantially stable peripheral lipoatrophy picture, and no osteopenia, as detected by a DEXA scan repeated 12 months after the first one, were found at our periodical clinical controls. This last drug choice (that of oral pioglitazone) shows how it may become cumbersome (but also very satisfactory for both caregivers and patients), to individualize, even to "tailor" the care of every HIV-infected patient according to his/her background, present problems, and future criticisms and strategies $[8,48,50,108]$. Among hypolipidemic drugs used for the concurrent, high-risk dyslipidemia in a patient with a major previous heart event, rosuvastatin was maintained at the same low dosage, due to its combined efficacytolerability issues just in high-risk HIV-infected patients undergoing cART [53,55,101,109], as well as an isolated adjunct of a titred dosage of $\mathrm{n}-3$ polyunsaturated fatty acids, which have been approved just in the prevention of major cardiovascular events [30,52,56,66], and preferred to fibrates in our case [29]. The "unavoidable" combined anti-HIV medications (cART), couldn't be stopped since mid-2008, but only simplified as far as possible [82], even though the virologic-immunologic target have been reached and have been maintained under complete control for a long term. With regard to acute or chronicprogressive loss of renal function in persons living with HIV and taking cART, tenofovir was the only known "intrinsically" nephrotoxic anti-HIV agent taken by our patient before its episode of acute kidney failure, but it was carefully re-challenged and proved perfectly safe in our case (until a 16-month follow-up period after the acute episode), and played its key role in both prior and subsequent triple "classical" cART regimens, even after the acute kidney injury episode [5,8,10,18,19,36,37-44,47,57$63,85,86,110]$.

The described episode of acute-onset kidney failure might have been favored by a broad spectrum of causes acting concurrently with HIV itself, and all underlying and overwhelming diseases, to lead at some point to a somewhat "critically" impaired renal activity [111], which may be followed by a po- 
tentially life-threatening "cascade" of events, such as metabolic acidosis and lactic acidosis plus hypokalemia and other potentially severe electrolyte and acid-base status imbalances [110,112], which are already well known adverse events of many HIV-associated medications [10,18,32,54,62], and may be prompted by the concurrent (ab)use of very common drugs, including trivial diuretics and some anti-hypertensive compounds, as well as oral antidiabetics like metformin, and NSAIDs at least. Just these insuline-sensitizing drugs are of extremely frequent use in the metabolic syndrome and related issues [48,50,113], where a sort of "vicious circle" is already of concern among metabolic alterations, insulin resistance, vessel and kidney injuries, pro-inflammatory cytokine cascade, an extensive and generalized "endothelitis", accelerated atherogenesis, finally followed by a global premature aging, characterized by an exponentially increased risk of developing life-threatening acute- or chronic-onset end-organ damages, like an "explosive" mixture, which is presently the major concern in people living with HIV [1,2,8,10,18,19,42,43,48,49,62,66,76].

In fact, the readers may easily imagine what will happen when all these related and unrelated conditions are becoming more and more common and severe, just among HIV-infected patients treated with cART, while these patients are increasing their mean age and their comorbidities, towards their elderly $[3-5,10,18,19,30,31,35,36,40-42,49,56,60,62,63,69,86]$.

\section{Differential diagnosis}

When trying to establish a differential diagnosis in a HIV-infected patient with an acute, sudden loss of kidney function while under cART, and concurrent diseases with their related, multiple medications, first of all we have to proceed as in the general population with a somewhat matched age, clinical and pharmacological background $[18,19,62,69,77,78,93]$. Therefore, we have to take into careful consideration all the endless concomitant conditions which may characterize or modify the kidney disease presentation and course, when HIV infection, comorbidities, antiretroviral drugs, and polypharmacy are of serious concern in age-comparable patients with some commonly encountered underlying diseases. Initially, it remains mandatory to detect whether the renal damage is primarily located in the renal glomeruli, or in the kidney tubules. Since abdominal ultrasonography studies are not expected to show relevant abormalities (as in our case), on the ground of a very trivial urinalysis (which did not show albumin or erythrocytes in the presented case), it is not wise that we are fronting a sort of "mainly" glomerular lesion (although our patient was already affected by a known essential arterial hypertension, and by a "more recent" major heart event, which represented the most relevant "clue" of a generalized, elevated risk of accelerated atherogenesis, possibly prompting other vital organ damages, as well as a "recently" diagnosed non-insulin-dependent but frank diabetes mellitus, requiring oral antidiabetic drugs, which also represent the worse "companion" of the metabolic syndrome, in terms of a frankly increased cardiovascular risk). As a consequence, already at the "glomerular" level, we wonder how many inter-reacting pathological conditions might have the kidney as the predestined target "victim" of such an "obscure conspiracy", which kept our patient under threat (Figure 1).

After ruling out a significant "glomerular" involvement on the ground of extensive clinical and laboratory examinations (a GFR of $83 \mathrm{ml} / \mathrm{min}$ was calculated upon patient's admission, together with a creatinine clearance of $103 \mathrm{ml} / \mathrm{min}$ ), a simple urinalysis, and a monitoring of urine output, subsequently it becomes appropriate to move immediately our attention on other causes and mechanisms which may affect the renal function through an injury at the "tubular" level.

When examining the differential diagnosis of our patient's acute kidney injury as a potential consequence of "tubular" more than "glomerular" damage, the clinical pathway must be approached using the "classical" nosology of "prerenal", "intrarenal", and "postrenal" causes [77,78]. A typical prerenal cause of acute kidney failure usually include the conditions where some cause of volume depletion is of concern. This could descend from multiple pathological conditions, and it may often be the "very trivial" consequence of an exaggerated diuresis, potentially due to a disproportionate loss of fluids, but 
Serum creatinine and standard urinalysis (for protein, glucose, blood search) to be performed at least quarterly, plus calculated glomerular filtration rate (GFR) (CD or MDRD estimates)

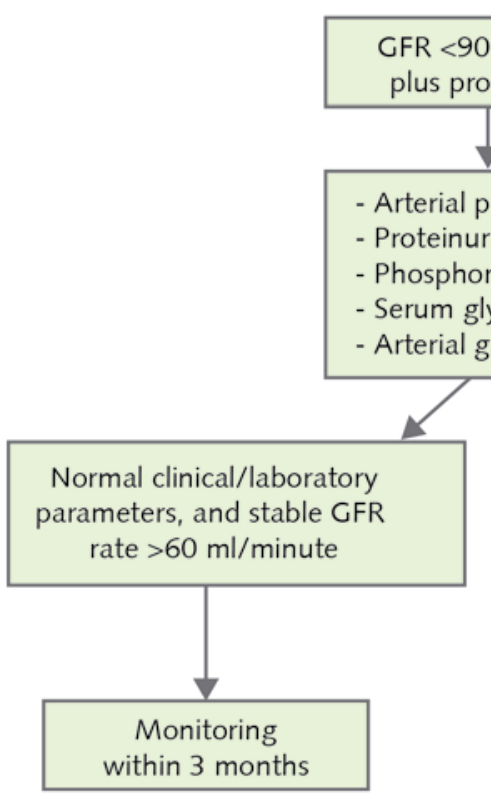

Proteinuria, or hyperglycemia, or hypophosphoremia, or ecographic abnormalities, or estimated GFR $<60 \mathrm{ml} /$ minute

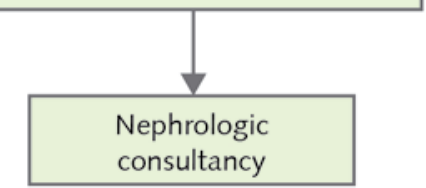

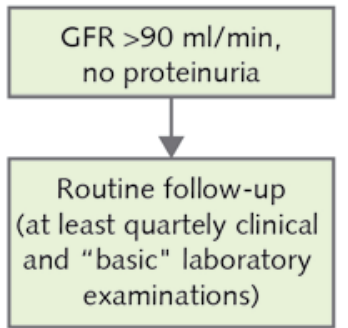

To be considered/excluded:

- HIVAN

- familiar history/modifiable risk factors

- major vascular "events"

- arterial hypertension

- metabolic syndrome

- diabetes mellitus

- aging-> increased comorbidities

- adverse drugs events (polipharmacy)

Figure 1. Standard monitoring of kidney function in HIV infected patients

it may be also due to both left and right heart ventricular dysfunction (extensively determined at the left side, but just in its initial stage at right heart ventricle in our patient, as assessed by a recent ultrasonography examination). But the origin may be "iatrogenic", of course:

- a simple, not-well tailored resort to long-term loop diuretics (with furosemide recently increased in its dosage, in our patient);

- the concurrent administration of slightly nephrotoxic drugs, like the recently introduced antihypertensive ACE-inhibitor and the oral antidiabetic drug metformin (as in our patient, too);

- on a long-term scenario, the virologically and immunologically effective and nephrologically "silent" tenofovir-containing very common cART regimen (the first-line fixed association of tenofovir-emtricitabine as the most prescribed antiretroviral backbone in the common clinical practice) $[10,18,62,82]$.

Based on the lack of initial, significant alterations of laboratory kidney examinations, and especially the urinalysis of our patient, an "intrarenal" cause of the acute failure would suggest to focus attention on the kidney tubules, at first. An acute tubular injury is caused by a severe cell toxicity, usually responsible of an equally acute tubular necrosis. The potential causes of a sudden, acute tubular necrosis in our case patient might include an extremely wide spectrum of conditions. After excluding the most common post-renal cause (the obvious bladder outlet obstruction), a pre-renal cause has to be seeked: a cART regimen including tenofovir should be the apparent "major" target for a proportional narrow knowledge of a HIV/AIDS or a clinical infectious disease specialist, also due the well known "intrinsic" kidney toxicity of tenofovir $[10,18,37,43,62,76,79,83,85]$, but... since "Internal Medicine" must be the founding ground of the entire Medicine, we have to consider carefully and promptly all the multiple concomitant conditions and medications, and we have also to look immediately for any possible kind of jatrogenic damage. Actually, a proportionally mild (but critical) volume depletion resulting in a proportional hypotension but without a repeated heart ischemic injury (excluded at first clinical exa- 
mination and ECG examination, as well as after serum troponin and heart enzymes testing), although relevant heart failure remnants were present and well documented in our case, as shown by an already severe, but stable left ventricle heart damage, and an initial right ventricle impairment, was detected at the last heart ultrasonography. In the mean time, the use of loop diuretics in association with ACEinhibitor antihypertensive agents and a very common antidiabetic agent like metformin (whose dosage had been just increased to reach the normal HB1c threshold), unadvertently led our patient to a sort of "blind alley".

So that finally, the somewhat occasional intake of a very common over-the-counter NSAID (i.e. ibuprofen, in our case), for an intercurrent, trivial back pain, became of major concern in our unfortunate case, when compared with all other "competing" causes of an acute renal injury, which added significantly one together with the other one, although every single drugs played a near negligible intrinsic kidney damage.

A quite remarkable laboratory finding in the patient's urinalysis, which deserves an enhanced value when re-reading carefully our case report, was the specific gravity tested at 1.009 . The lowest threshold of specific gravity of 1.005 usually indicates the most diluted urines, while a specific gravity of 1.030 represents the most concentrated ones. When other renal lesions are excluded (or played a non-significant role, as in our case), the kidneys in their attempt to preserve both intravascular volume and serum sodium levels do excrete concentrated urines (up to a specific gravity of 1,030). The value observed in our patient (i.e. 1.009) is therefore named isosthenuria, since urine concentration is within normal limits. The presence of an isosthenuria reflects tubules that have neither concentrated nor diluted the urine, but this issue occurs in a very "critical" patient with a correct fluid balance (as assessed by the urgent, laboratory examinations available at that time at our Day-Hospital facility), but rapidly shifting towards a potentially life-threatening metabolic acidosis and hypokalemia, followed by a precipitous worsening of kidney function abnormalities in the early hospitalization period, luckily corrected by the prompt detection of a severe metabolic acidosis of renal origin, and timely monitorized and treated as appropriate. As a consequence, when in the clinical setting of an acute kidney injury, it is mandatory to exclude or confirm a phenomenon of acute tubular necrosis, and its expected consequences.

When considering the "last straw of a pot already filled to the brim", in our "especially unfortunate", but finally even "lucky" patient, a major role has been probably played just by the most commonly used drugs all over the world, i.e. the NSAIDs, which are well known as prescribed but (even more...) as over-the-counter and even more self-prescribed medications burdened by non-negligible toxicity [114], and sometimes used also by patients with suicidal behavior [115], similarly to an episode due to a probable long-term repeated prescription (a sort of "self-prescription" of immunosuppressive drugs with lethal outcome), which was described by us five years ago [98].

In the field of potential nephrotoxicity due to NSAIDs [93,114], we have to consider the primary mechanism of action of these drugs: they substantially blunt the synthesis of prostaglandins (i.e. the major elements of inflammation as a whole), but also lead to a concurrent action on vasoconstricting hormones, such as angiotensin in the renal scenario. The expected consequence of decreasing kidney perfusion becomes more evident among the different kinds of volume-depleted patients, through adjunctive mechanisms of either vasodilation, or impaired/reduced heart function, which leads to a further reduction of the perfusion pressure. These subjects actually "rely" on their prostaglandins all day round, in order to try "manage" their renal perfusion as far as possible. When prostaglandins are inhibited by NSAIDs (and similar drugs) for example, the renal function of individuals with an arterial volume depletion becomes proportionally volume-sensitive: at this "delicate" time, very subtle or near negligible, and apparently "minor" changes in global volume may become responsible of large decreases of renal perfusion. Like the events due to an absolute volume depletion (easy to rule out in our case report), the decrease in perfusion at some critical point is expected to result in a transition phase, from 
the kidney "asking for sodium" and conserving the volume, to an apparently sudden and unexpected kidney "displacement", when an impaired perfusion reaches a critical threshold, and finally turns into the occurrence of a true, full-blown tubular cell necrosis, and immediately thereafter in an acute kidney injury [77,78,93]. As perfectly known by everybody in the field of Internal Medicine, the concurrent administration of a common ACE-inhibitor antihypertensive like enalapril (taken at standard dosage by our patient, before and after his acute renal "crisis"), and a similar common resort to furosemide and metformin, further "confuse" this already cumbersome scenario (if possible), by making the patient even more and more susceptible to otherwise minimal volume changes.

As anticipated, nephrotoxicity is a well known but quite uncommon and usually reversible complication of tenofovir administration for both HBV and especially HCV chronic infection [19,37-39,43$45,60,69,76,85,86,93]$, which may possibly be prompted when other anti-HIV nucleos(t)ide analogue (i.e. didanosine) [38], and maybe when the anti-HCV ribavirin [69,89] are co-administered.

Actually, the renal spectrum of adverse effects of the "intrinsically" nephrotoxic tenofovir [10,18,19,37,39, 40,43,47,57-60,62,63,76,79,83,85,86], classically includes:

- the potentially serious but reversible Fanconi's syndrome, similar to inherited or other diseaserelated (especially malignancy-related) multiple end-organ dysfunctions [116], characterized by an excessive tubular loss of glucose and/or electrolytes and/or albumin, and associated with hypokalemia and hypophosphoremia) $[10,37,39,85]$;

- infrequent, mostly anecdotal cases of acute kidney failure (like ours), resembling Fanconi's disease in their clinical onset, presentation, and outcome [10,18,19,38,40,57,59,60,62,76,85,86]; in fact, the renal alteration completely recovered, and the re-challenge with another tenofovir-containing cART regimen proved safe for the entire follow-up period;

- also extremely rare cases of diabetes insipidus, of renal origin obviously [39].

To complicate this already cumbersome clinical picture, we have to remind that these rare toxicities have been reported within one month and up to 15 months after the initiation of a tenofovir-including therapy for whatsoever indication $[10,18,47,62,63,69,76,81,82,84]$. Anyway, both observational studies and registrative clinical trials estimate the rate of impairment of renal function among patients receiving tenofovir to be approximately $1 \%$ of cases, mostly reversible in a limited temporal span $[10,18,19,38,47,57-60,62,63,76,84-86]$.

However, the pathogenetic pathway by which tenofovir may be linked to an acute kidney injury, other than by an histopathological demonstration of the so-called "kariomegaly" [59], has not reached to the best of our knowledge. As a trivial deduction, and because of the infrequent availability of systematic histopathological examinations of these anecdotal cases, it becomes possible that an elevated proportion of acute kidney injuries involving patients on tenofovir do not include tenofovir itself as the "major" causative agent, as previously anticipated.

Even though the risk factors for these proportionally uncommon kidney toxicities are still not perfectly estimated in their frequency and known in their "crossing" and "shared" pathogenetic mechanisms $[10,18,19,38,44,45,57,59,60,62,63,71,76-78,85,86,93,94]$, data extrapolated from randomized clinical trials, small case series, and anecdotal reports have suggested that probably a broad series of factors may play a role in supporting this acute condition in some selected subjects, burdened by several, easily recognizable risk factors $[10,19,59,69,77,78,85,93]$ :

- an already existing impairment of renal function (which was absent in our case, but may be prompted by an accelerated atherogenesis, a pre-existing arterial hypertension, the overwhelming acute heart infarction and the diabetes mellitus of novel diagnosis);

- a lower body weight (it was not the case of our patient, too);

- a lower absolute $\mathrm{CD}^{+} \mathrm{T}$-lymphocyte count (also absent in our otherwise unfortunate patient, during all his entire follow-up period); 
- the concomitant resort to other "severe, intrinsic" nephrotoxic antiretroviral medications, with regard to the antiretroviral nucleos $(\mathrm{t})$ ide analogue didanosine [38], and the well-known "intrinsically" nephrotoxic protease inhibitor indinavir $[10,44,45,93,94]$, as well as other antimicrobial agents of extensively common use among HIV-infected patients (i.e. ribavirin, adefovir, ganciclovir, cidofovir, foscarnet, aminoglycosides, amphotericin B, pentamidin, vancomycin, teicoplanin, interleukin-2, and many others), but our patient never received these drugs in his proportionally "recent" history of asymptomatic HIV disease, so that he never underwent treatments with other frankly nephrotoxic compounds $[7,8,10,18,19,25,59,61,62,68,69,80,81,89]$, and did not suffer of chronic hepatitis B or C, which are frequent events in HIV-infected individuals [28,69,89-92].

With regard to the second of the four identified risk factor, a missed renal impairment affecting for example a person with a reduced skeletal muscle mass may result in a potential mediator of even severe nephrotoxicity. That is to say, a serum creatinine value of $1.1 \mathrm{mg} / \mathrm{dl}$ in an around 60 -year-old female patient with a body weight of $60 \mathrm{~kg}$ would result in a calculated creatinine clearance of slightly more than $50 \mathrm{ml} / \mathrm{min}$. In this example, failure to recognize promptly "borderline" serum creatinine levels of around $1.0 \mathrm{mg} / \mathrm{dl}$, might lead to the administration of a disproportionally elevated tenofovir dosage, and finally to an increased risk of renal function impairment [10,18,19,37-40,60,62,85,86].

The expected (and repeatedly announced previously), potential contributors to tenofovir-related renal toxicity include the physician's prescription of NSAIDs, or the "self-prescription" of over-thecounter NSAIDs [93], as well as multiple concurrent medications including antiretrovirals themselves, antimicrobial agents, and all the "politherapy" used to manage the adverse events of cART itself $[5-7,10,18,19,36,37-43,46,54,57-62,76,85,86,92]$, as well as the role played by the concurrent immune recovery in supporting "paradoxically" greater immune-mediated pathogenetic mechanisms $[22,63]$. NSAIDs are well known and very widely administered pharmacologic agents, which are burdened by risks of inducing an acute kidney failure [93], even independently of the concomitant employment of other nephrotoxic drugs. Small but significant case series demonstrated that acute kidney failure may occur in patients who concomitantly receive both tenofovir and NSAIDs [10,18,19,43,60,76,93].

As a matter of fact, from a clinical point of view the still incomplete pathogenetic pathway of all drugdrug associations capable to "fire up" an acute renal damage in these complicated-to-manage patients $[10,18,19,44,45,62,63,76-78,93]$ does not change the "bedside" management in the field of medicine, which plays a critical role in its promptness, but does not need specific measures according to the majority of potentially involved drugs. In fact, after a timely diagnosis (plus a very basic kidney ultrasonographic assay), and thanks to a prompt and adequate delivery of supportive care, the great majority of these acute cases recover spontaneously.

The problem remains regarding how to move in the next future: in our specific case, it was somewhat diffucult to answer the question whether tenofovir should be re-introduced or not, after complete recovery of an episode of acute kidney injury prompted by multiple, and continuously "moving" and correlated and overlapping causes and medications. Of course, during the acute phase of an acute renal failure of still uncertain origin, all drugs which are expected to induce or worsen renal impairment, together with all medications and "recreational drugs" which that are mainly cleared by the kidney, have to be immediately discontinued, as a first prudential measure [18,19,62,63,67,77,78,93].

Furthermore, since we still cannot weigh how elevated is the incremental risk associated with the concurrent administration of all the above-mentioned drugs in combination with each other, when giving always a strict priority to patient's safety, the caregivers of all patients with HIV disease who are receiving tenofovir (and also those with chronic hepatitis B treated with tenofovir, more than the "cousin" low-dosage and less nephrotoxic adefovir) $[10,80,81]$ should keep their best careful consideration on how to manage trivial, intercurrent conditions like inflammation and pain, in patients under continued tenofovir therapy for their potentially severe, chronic viral diseases, like HIV and HBV infection, and related disorders and comorbidities. 


\section{Conclusions}

Our case report is a contemporary "portrait", which is a truly representative figure of a subject living with HIV and successfully continuing his well tolerated cART regimen, just in the years 2010-2011 (i.e. 27 years after the discovery of HIV as the agent of AIDS) [117].

Incidentally diagnosed with an asymptomatic, heterosexually-transmitted HIV disease together with a concurrent latent syphilis, our patient was already in his fifties, and had some familiar and lifestyle "stigmas" which are very common in our general population, and even more in the general population of HIV-infected patients, which is becoming more and more older due to two apparently opposite phenomenons: the previously diagnosed subjects living with HIV now reach their elderly thanks to cART, while we have a growing number of individuals with a missed or neglected HIV disease until their elderly [3-5,18,25,30,31,35,36,40-42,56,60,62,63,69,86].

Our exemplary patient obtained a rapid, complete, and sustained virological response to his early and subsequent cART regimens, whose five changes were all due to tolerability/convenience issues, in absence of any failure and viral resistance, as expected due to the present availability of over 20 antiretroviral compounds, and multiple first-choice drugs belonging to many antiretroviral classes $[10,82]$. Moreover, when compared with his several underlying and overwhelming disorders, no HIV-related pathologies (including very minor and indirectly-related ones) were detected at any time during a yearly clinical follow-up, save mild, transient, and self-limiting adverse events due to some cART regimens (i.e. CNS disturbances and rash attributable to efavirenz introduction, and later hyperbilirubinemia due to the typical metabolic-elimination pathway of the HIV protease inhibitor atazanavir) [82]. Taking into account of the increased (and increasing) life expectancy of patients living with HIV (now approximating that of the general population), it is more and more necessary to consider adequately all the long-term consequences of cART and underlying, also HIV-unrelated disorders, when managing HIV-infected patients who cannot anymore stop or interrupt their cART treatment $[82,118,119]$, due to the documented increase of early life-threatening events observed in a short time after therapy discontinuation, and probably mediated by a cytokine cascade prompting cardio- and cerebrovascular events, and also end-organ failures, which are caused by the same vicious circle which is primarily caused by the renewed replication of HIV $[1,2,66,82,118-120]$, although the conclusions emerging from some multi-cohort and post-hoc analyses should be considered carefully [121,122], when translated into daily clinical practice, and especially when incorporated into the updated guidelines for HIV disease management [82], which become the reference aid for drug prescription worldwide.

Among end-organ (vital organ) toxicities, the kidney toxicity must be carefully assessed, especially in patients with a history of cardiovascular or renal disease itself, those exposed to nephrotoxic drugs presently or in the past, patients with comorbidies involving the kidney, or patients who are simply over their fifties $[3-5,8,10,30,31,35,36,40,43,56,60,78,86,93]$, as our representative case report tells.

Furthermore, our case report recalls and underlines the multiple mechanisms by which various medications may interact in the strictest definition of the term, may play a negative, additive or synergistic toxic effect towards one or multiple organs and tissues, or result in a trivial rise of some (apparently) isolated kidney function parameters, as an early clue of a slow- or rapid-onset kidney toxicity, but may also represent the very initial sign of an acute kidney failure, or that of a progression towards a chronic renal failure when a pre-existing renal impairment is already present or has been neglected or missed in the past. As expected, both acute and chronic kidney failure are life-threatening conditions, in their potential short- and long-term evolution, through multiple and often not necessarily related pathogenetic pathways $[3-5,10,18,30,31,35,36,41,42,56,60,62,63,69,86]$.

Since the patients living with HIV are going to reach the same life expectancy of the general population, but are prone to a sort of "accelerated aging" (also due to multiple, often unrelated mechanisms) $[2,5,8,9,36,42,69,123]$, therefore they are expected to develop worsening comorbidities, and these sce- 
narios are expected to become increasingly common and intricated, and will come to the attention of specialists other than infectious diseases ones, in their progressive steps of their multiple underlying or concurrent morbidities. From the viewpoint of a multidiscliplinary, "super-specialistic" appraisal to these patients $[8,10]$, we underline the need of a careful monitoring of renal function in all patients living with HIV, and especially in those treated with cART.

In stable, HIV-infected patients undergoing cART therapy without any relevant comorbidities and comedications, this monitoring should be started at least with a longitudinal assessment of glomerular filtration rate (GFR), creatinine clearance, and urine protein and electrolyte contents. Otherwise, the rare, but life-threatening episodes of metabolic or lactic acidosis, which are associated with a concurrent, severe impairment of renal function and significant electrolyte imbalances, should be recognized and managed as early as possible $[5,6,8,10,32,33,36,54]$. In stable patients, as in the general population, the GFR test is much more accurate, when compared with the less sensitive serum creatinine levels, in monitoring the kidney function, and even more to catch the very early and mild abnormalities of renal function which may precede a full-blown chronic (but also a sudden and acute) kidney dysfunction, as happened in our case. A tenofovir-related nephrotoxicity should be addressed when we observe a reduction of GFR exceeding 50\%, and/or when serum creatinine clearance levels drop of $25 \mathrm{ml} / \mathrm{min}$ or more. We retrospectively did not find any significant variation of both kidney function parameters, when comparing the values detected during hospitalization, with all the available quarterly outpatient clinical controls of our patient. However, when selecting and applying the GFR calculation in the estimated prediction of renal function, we have to take into careful account both age and gender, and also the race (for the Modification of Diet in Renal Diseases - MDRD - equation), while we have to consider the body weight (for the Cockcroft-Gault - CG - equation). Significant distortions of GFR measurements are expected to occur, despite substantially similar serum creatinine values, should all these variable are not accounted for [124,125].

Regardless of the main (or predominant) etiology of an eventual, acute kidney injury, in subjects living with HIV as well as in the general population matched for age, gender, and race, an appropriate diagnostic and therapeutic pathway should include:

- the assessment, the management, and the removal (if possible), of all risk factors, whether primary, secondary, or potential in origin, along with the provision of a prompt and appropriate supportive care, should an acute problem is of concern. Some causes of acute renal failure can be diagnosed through trivial blood tests, such as rhabdomyolysis using serum creatinine phosphokinase and myoglobin levels $[33,77,78]$, which may be performed on "urgent" basis at our outpatient facilities, as opposed to urine electrolyte levels and serum electrolytes other than sodium, potassium, and calcium, which require a specific over-the-phone contact between the physicians in care and the Central Laboratory of our Hospital, based on clinical suspicion, and a 24-hour collection and storage of urine, which is not applicable in very urgent circumstances;

- unfortunately in most cases of acute-onset renal failure the initial trigger factor cannot be identified, or depends on multiple, associate conditions (as in our representative patient). A renal biopsy will demonstrate some non-specific cellular damages characteristic of acute kidney injury, and may help in ruling out an interstitial nephritis, which has been also recognized as a potentially severe renal toxicity prompted by cART $[18,63,108,126]$. However, also histopathological studies cannot allow us to identify the primary or the direct cause of a sudden kidney function failure, in the large majority of cases $[77,108,127,128]$. However, from a strictly clinical and management point of view, the specific identification of the source and the pathological picture are not required to physician in charge of such a medical emergency, given that non-specific supportive (although intensive) monitoring and treatment are always indicated, as a matter of urgency. In the reported patient of ours, the initial discontinuing of tenofovir was appropriate not only because of potential role of this drug in causing a (rare) acute-onset kidney failure (similar to Fanconi's disease in its clinical features), 
but also because of the drug's prolonged half-life, which is expected during a potentially severe renal insufficiency $[10,18,43,62,63,76,79,83]$. In addition, it seemed essential to discontinue immediately the anti-hypertensive ACE-inhibitor drug [128], the loop diuretic furosemide, obviously the NSAIDs $[10,18,19,43,60,76,93]$, and maybe metformin too [129,130], which may be associated with severe lactacidemia, regardless of its intrinsic renal safety profile [130]. The therapeutic approach is focused on providing a well "tailored" intravascular fluid intake and control, and to re-calibrate all fluid and electrolytes delivery on the basis of a continuous clinical and laboratory monitoring.

- an extremely careful attention to all kidney function parameters, i.e. at least creatinine clearance, GFR, urinalysis, all possibly serum and urine electrolytes (especially serum potassium and phosphate levels, as in our case) should be ensured and monitorized at least every 2-3 months to all patients taking tenofovir for either HIV or chronic HBV infection, when they are fully stable from a clinical point of view. A tenofovir-related nephrotoxicity should be addressed when we observe a reduction of GFR exceeding $50 \%$ of previously checked levels, and/or when serum creatinine clearance values drop of $25 \mathrm{ml} /$ minute or more: it was not the case of our patient, whose glomerular function remained substantially preserved, despite his already treated arterial hypertension, the acute myocardial infarction with a reduced left ventricle injection fraction, and his revent, overwhelming diabetes mellitus. On the other hand, when an acute-onset kidney failure or a sudden, unexpected reduction of kidney function occur, current laboratory testing should be performed on an emergency basis, together with an arterial blood gas analysis, and serum lactic acid, and all serum and urine electrolyte determination: in fact, an unexpected hypophosphoremia may prompt a more severe kidney dysfunction in patients receiving tenofovir versus abacavir as their nucleos(t)ide analogue backbone [87]. In a situation of acid-base derangement like that of our critically ill patient, urinary density measurement proved useful to assess the disorders of water balance and to discriminate between prerenal azotemia and acute tubular necrosis, and to prompt the immediate resort to supportive life measures, and more sophisticated laboratory examination, carried out as soon as possible.

- in the understanding of the intricated pathogenetic and clinical pathways potentially involving the kidneys and their function (including the genetic determinants of multiple possible kidney function alterations, which cannot be explored in the current clinical practice) [74,75], it is mandatory for all caregivers of HIV disease and all specialists involved with their consultancy, to be aware of the management of multiple comorbidities together with HIV (and/or HBV) infection, which are becoming more and more easy to be controlled (HIV infection) or cured in the majority of cases (chronic hepatitis B), in their well established virological, immunological, clinical and instrumental parameters, when compared with all the emerging problems in the field of "polypharmacology" used daily to manage concurrent diseases, or even to blunt expected cART toxicities, for example, which are becoming a major target of attention of all clinical infectious diseases specialists, since over 15 years, just the era of the so-called "higly active antiretroviral therapy" or HAART, presently called cART [1,3,5,10,13-15,18,19,28-33,35,36,46,52,53,55,56,62,103].

- when considering the underlying, antiretroviral therapy [82], which cannot be interrupted but only simplified due to an increased risk of disease progression or other life-threatening disorders [118120], we have to consider that the different cART regimens are well characterized by a proportionally different renal metabolism and excretion, and this issue must addressed in order to "tailor" the best therapeutic choice of cART, in patients who are at risk of developing renal impairment, had a prior episode of renal toxicity (as in our case), or may experience a worsening of their pre-existing chronic renal disease $[8,10,18,19,62]$. As anticipated, an increased risk of a progressive decline of kidney function over a proportionally prolonged time span (as opposed to the above-mentioned acute kidney failure of our patient) has been observed in HIV-infected patients treated with tenofovir as a part of the nucleos $(\mathrm{t})$ ide backbone (especially with the non-recommended use with didanosine) [38], and the negative pharmacokinetic interactions with abacavir, which did not show 
an in vivo additive effect to tenofovir [131]. However, when selecting a "traditional" nucleos(t)ide analogue backbone for our patient, abacavir-containg combinations have been excluded after a careful, collegial discussion, since:

- our patient already experienced an allergic reaction to efavirenz, and we are aware that some hypersensitivity reactions to abacavir may occur also in the absence of the specific genotyping testing [71,72];

- moreover, abacavir administration has been linked in some studies (but it led to a fierce, endless discussion according to other data, which primed the so-called endless "abacavir saga") [122, $123]$ to an overall increased, global cardiovascular risk $[119,120]$, so that it was "probably" to avoid in our patient, who suffered of a recent acute heart infarction, even though the two available fixed dose combinations tenofovir-emtricitabine and abacavir-lamivudine, proved a similar virological activity in both antiretroviral naïve and experienced patients $[47,82,87,132,133]$, although patients on a tenofovir- versus abacavir-containing regimen showed an increased risk of kidney dysfunction, as recently underlined in the "ASSERT" study, which addressed just serum hypophosphoremia as a serious "clue" of an incipient renal toxicity in patients taking tenofoviremtricitabine, as opposed to those treated with abacavir-lamivudine, with all enrolled patients taking efavirenz as the "third" drug of their cART regimen [87].

- however, our 16-month-long "re-challenge", carried out successfully just with the same fixeddose tenofovir-emtricitabine combination, confirms the safety of tenofovir, also after an acute, but reversible kidney function damage prompted by some other concurrent toxicity factors [10 $, 18,19,38,40,57,59,60,62,76,85]$.

- on the other hand, we have plenty of data regarding the safe use of a "classical" triple cART containing a tenofovir-based background, plus either a non-nucleoside reverse transcriptase inhibitor, or a boosted protease inhibitor, as the so-called "third drugs". As known, when excluding indinavir, nelfinavir, and unboosted fosamprenavir and atazanavir, all other available HIV protease inhibitors need a variable dosage of ritonavir boosting [82]. Regardless of ritonavir booster dosage, all available protease inhibitors may non-negligibly increase the kidney toxicity of a tenofovir-containing cART, even though this phenomenon becomes clinically relevant only when other underlying diseases, supporting factors, and polypharmacy are of concern $[10,18,19,37,38-41,43-45,47,60,62,63,69,71,76,85,86,88,93,94]$. The majority of authors reported a negligible, but not unsignificantly increased toxicity at the renal level when patients receiving a tenofovir-containg backbone have been compared with those who were treated with tenofovir with either emtricitabine or lamivudine, together with a non-nucleoside reverse transcriptase inhibitor at a some extent $[10,47,71,88]$, or those who took a cART which did not include tenofovir at all $[10,40]$. To add even more confusion, some preliminary findings were not supported by the figures obtained in the "HOPS" study: in this case, the concomitant administration of tenofovir and a boosted protease inhibitor did not show relevant changes of kidney function, versus HIV-infected patients receiving tenofovir alone [7,41]. Since the a robust evidence is not available to prompt avoidance of concurrent use of a boosted protease inhibitors together with tenofovir (a very common cART regimen, in our daily clinical practice, as recommended by the present guidelines of HIV treatment $[82,88]$ ) an increased surveillance of kidney function in patients who undergo all these agents concomitantly seems absolutely needed, as well as the attention of infectious diseases and HIV/AIDS specialist, added to that of specialist consultants (especially nephrologists, cardiologists, and diabetologists, as in our case).

In our special case report, waiting for a large employment of nucleos(t)ide sparing cART regimens and that of regimens completely relying on agents other than nucleos(t)ide and non-nucleoside analogue reverse transcriptase inhibitors, which are still not so widely employed in patients showing an excellent and sustained response to other cART regimens [82], due to their still incomplete indications and often 
elevated costs [11,133-135], we had to select which was the most effective, and "comfortable" classic third agent for our patient. Since we were forced to eliminate immediately all available non-nucleoside reverse transcriptase inhibitors due to the former adverse cutaneous and CNS reaction to efavirenz, and a large portion of HIV protease inhibitors remained fully effective to our unfortunate patient, who never failed a cART regimen in his prolonged follow-up, in order to accompany the finally maintained tenofovir-emtricitabine nucleos(t)ide backbone, we selected the effective, safe, and convenient darunavir (at $800 \mathrm{mg}$ once daily) plus a minimum ritonavir booster dosage (100 mg), after considering that the acute kidney event occurred under fosamprenavir-ritonavir combination, the atazanavir-ritonavir association was not tolerated due to a trivial but persistant hyperbilirubinemia, lopinavir-ritonavir has a well known unfavorable dyslipidemic profile, and novel profiles of risk are at the horizon in patients receiving protease inhibitor-based cART regimens [136], but may be controlled by statins, too, especially when a patient at very elevated risk of repeated major vascular events is of concern. As known since many years, the HIV protease inhibitors may be easily "sequenced" on both a tolerability and an efficacy point of view [137-139]. The intrinsic renal safety of darunavir itself and the smallest required dosage of ritonavir $(100 \mathrm{mg}$ only), also when combined with tenofoviremtricitabine [140], is expected to outweight the already modest risk of adjunctive toxicity at the renal level which remains a shared feature of all HIV protease inhibitors [10,18,19,37,39,43$45,60,62,63,71,76,85,86,88,93,94]$, together with the slightly increased cost of this last protease inhibitor, which has been recently approved also for first-line naïve patients, and in its once-daily dosing. However, in these patients with previous, current and future risk of end-organ injury, the raising funding and pharmacoeconomic issues should leave the place to a fair, patientoriented, ethical approach to medicine $[22,27,50,73,141]$.

Among factors which are known to predict acute-onset or to worsen a pre-existing kidney toxicity, a previous experience with any kind of cART act negatively by itself, and especially when well-known
Take-home messages and questions

for further researches

- Pay maximum attention to "all" drugs and drug combinations with a renal metabolism and/or excretion

- For HIV-infected patients already experienced with any CART regimen, this condition poses some increased risk of kidney and other end-organ toxicity "per se"

- The mechanism of kidney toxicity may be different, according to the different drugs concurrently administered just to patients living with HIV, as opposed to the general population, such as:

tenofovir

indinavir

didanosine

ribavirin

protease inhibitors (with focus on ritonavir as the commonly used booster)

- Extreme caution should be applied, when associating other compounds to CART regimens, with special reference for a series of antimicrobial agents, which are of frequent use just among HIV-infected patients, like:

previously or concurrently administered antiretroviral agents, especially indinavir and tenofovir, but also boosted HIV protease inhibitors

adefovir

aciclovir and ganciclovir, and their derivatives

cidofovir

foscarnet

pentamidine

aminoglycosides

amphotericin B and its lipid derivatives glycopeptides

interleukin-2

- Also to be considered when assessing individual HIV-infected patients:

personal and familiar history for hypertension of any origin, whatsoever renal disease, cardiovascular disorder, insulinresistance, and diabetes mellitus

lifestyle (job, diet, cigarette smoking, physical exercise, etc.) acute or incidental or chronic pain (prompting the unadvertent use of prescription and over-the-counter NSAIDs) 
nephrotoxic agents have been administered in the past, especially for a proportionally prolonged time. Some practical questions regarding the management of HIV-infected patients undergoing cART are briefly summarized in the box. An increased time between tenofovir administration has been recommended in subjects with a severe kidney and also liver function impairment, where the potent antiviral activity of this compound has been successfully exploited also in these difficult-to-treat patients [142]. To conclude, a strategic approach to HIV infection management should enable all individuals living with HIV to aspire to a long life expectancy, with minimized end-organ compromise caused by both the virus, the cART, and underlying or concurrent diseases and/or treatments.

Only a truly multidisciplinary team will be the best possible answer to these emerging and potentially life-threatening conditions, and the experiences shared between different Specialists will enrich both scientific and clinical expertise of everyone engaged to take care of HIV disease in its ever complicating scenario. This intricated situation, after the initial difficulties substantially confined to the proportionally "narrow" point of view of each involved Specialist (with Clinical Infectious Diseases Specialist ranking first), is expected to become a very valuable resource, and a "real life", ever moving experimental laboratory, and also a "training ground", and a "work in progress" for Clinical Infectious Diseases caregivers, and all the Consultants Specialists who share the care of HIV-infected patients, which is becoming more difficult to manage, just when we have over 20 available anti-HIV drugs belonging to over six well developed pharmacological and therapeutic classes, depending on their mechanism of action against HIV: i.e., nucleoside reverse transcriptase inhibitors, non-nucleoside reverse transcriptase inhibitors, protease inhibitors, integrase inhibitors, fusion inhibitors, entry inhibitors), plus more and more antiretroviral drugs in their pipeline [143].

The "adventure" of HIV disease management and treatment, which started 30 years ago, in 1981 [131], had its first historical step at the time of viral recognition by Luc Montagnier and Robert C. Gallo in the year 1983 [117], then gained a subsequent step thanks to the possibility to measure plasma viral load as a major virological marker of disease progression monitoring, until we had got the first "triple therapies" (HAART) available, since the mid-nineties [144,145], is now becoming more and more fascinating, in its continued evolution concurrently with that of the available diagnostic and therapeutic resource, which have been discovered and developed by human beings since the year 1981, I their fight against HIV, which is now "entering its fourties", so that a finally adult age.

\section{The review in brief}

Clinical question Revise and discuss causes of renal failure in HIV patients, with special attention to interactions between antiretroviral therapy and the even growing exposure to multiple different drug and drug classes

Type of review Narrative

Search of the Medline search for English-language articles using the following keywords: HIV infection, antiretroviral literature treatment, tenofovir, acute renal failure, comorbidities, toxicity, drug surveillance

Conclusions A multidisciplinary vision and approach is mandatory for the diagnostic, clinical, and therapeutic management of kidney disorders occurring in patients living with HIV

Limitations The evolving subjects treated in this review deserve a continued update of informations coming from the international literature, with special reference to novel antiretroviral drugs approved for the management of HIV disease, and their potential (direct and indirect) interaction with kidney function 


\section{Acknowledgement}

The Authors wish to thank Dr. Liliana E. Weimer of the "Istituto Superiore di Sanità" (ISS), Rome, Italy, for providing her kind advice in drafting the manuscript.

This case represents the key point for a debate around the increasing frequency of "polypharmacy" in the field of HIV infection, even when HIV resistance to antiretroviral is not a concern.

\section{References}

1. Calza L, Manfredi R, Pocaterra D, et al. Risk of premature atherosclerosis and ischemic heart disease associated with HIV infection ad antiretroviral therapy. J Infect 2008; 57: 16-32

2. Calza L, Pocaterra D, Pavoni M, et al. Plasma levels of VCAM-1, ICAM-1, E-Selectin, and PSelectin in $99 \mathrm{HIV}$-positive patients versus $51 \mathrm{HIV}$-negative healthy controls. J Acquir Immune Defic Syndr 2009; 50: 430-2

3. Manfredi R, Mastroianni A, Coronado OV, et al. Hyperuricemia and progression of HIV disease. J Acquir Immune Defic Syndr Hum Retrovirol 1996; 12: 318-9

4. Manfredi R, Calza L, Cocchi D, et al. Antiretroviral treatment and advanced age: epidemiologic, laboratory, and clinical features in the elderly. J Acquir Immune Defic Syndr 2003; 33: 112-4

5. Manfredi R. HIV infection and advanced age: emerging epidemiological, clinical, and management issues. Ageing Res Rev 2004; 3: 31-54

6. Schwartz EJ, Szczech LA, Ross MJ, et al. Highly active antiretroviral therapy and the epidemic of HIV+ end-stage renal disease. J Am Soc Nephrol 2005; 16: 2412-20

7. Palella FJ Jr, Baker RK, Moorman AC, et al. Mortality in the highly active antiretroviral therapy era; changing causes of death and disease in the HIV outpatient study. J Acquir Immune Defic Syndr 2006; 43: 27-34

8. Rockstroh J, Guaraldi G, Deray G. HIV and the body: a review of multidisciplinary management. HIV Med 2010; 11 (Suppl. 2): 1-8. doi: 10.1111/j.1468-1293.2010.00859.x

9. De Gaetano Donati K, Rabagliati R, Tumbarello M, et al. Increased soluble markers of endothelial dysfunction in HIV-positive patients under highly active antiretroviral therapy. AIDS 2003; 17: 765-8

10. Cooper RD, Wiebe N, Smith N, et al. Systematic review and meta-analysis: renal safety of tenofovir disoproxil fumarate in HIV-infected patients. Clin Infect Dis 2010; 51: 496-505

11. Ghosh RK, Ghosh SM, Chawla S. Recent advances in antiretroviral drugs. Expert Opin Pharmacother 2011; 12: 31-46

12. Manfredi R, Pieri F, Pileri SA, et al. The changing face of AIDS-related opportunism: cryptococcosis in the highly active antiretroviral therapy (HAART) era. Case reports and literature review. Mycopathologia 1999; 148: 73-8

13. Manfredi R, Chiodo F. Features of AIDS and AIDS defining diseases during the highly active antiretroviral therapy (HAART) era, compared with the pre-HAART period: a case-control study. Sex Transm Infect 2000; 76: 145-6

14. Manfredi R, Calza L, Chiodo F. Three to seven concurrent AIDS-defining disorders at first hospitalization of AIDS presenters as an unexpected emerging feature during the era of highly active antiretroviral therapy. AIDS 2002; 16: 2356-8

15. Manfredi R, Calza L, Chiodo F. Emerging of dual AIDS associated neoplastic diseases in the era of highly active antiretroviral therapy. Sex Transm Infect 2003; 79: 345-6

16. Manfredi R, Marinacci G, Calza L, et al. Diffuse cutaneous dissemination of visceral leishmaniasis during human immunodeficiency virus (HIV) infection, despite negligible immunodeficiency: 
repeated failure of liposomal amphotericin B administration, followed by successful long-term pentamidine and paromomycin administration. Int J Antimicrob Agents 2008; 31: 590-2

17. Manfredi R, Calza L, Chiodo F. Dual Candida albicans and Cryptococcus neoformans fungaemia in an AIDS presenter: a unique disease association in the highly active antiretroviral therapy (HAART) era. J Med Microbiol 2002; 51: 1135-7

18. Jao J, Wyatt CM. Antiretroviral medications: adverse effects on the kidney. Adv Chronic Kidney Dis 2010; 17: 72-82

19. Campbell LJ, Ibrahim F, Fisher M, et al. Spectrum of chronic kidney disease in HIV-infected patients. HIV Med 2009; 10: 329-36

20. Calza L, Manfredi R, Mastroianni A, et al. Osteonecrosis and highly active antiretroviral therapy during HIV infection: report of a series ad literature review. AIDS Patient Care STDS 2001; 15: 385-9

21. Manfredi R, Battista G, Sassi C, et al. Morphofunctional evolution of thymus response after firstline combined antiretroviral therapy in adult HIV-infected patients. Med Malad Infect 2003; 33: 584-9

22. Elston JW, Thaker H. Immune reconstitution inflammatory syndrome. Int J STD AIDS 2009; 20: 221-4

23. Manfredi R, Calza L, Chiodo F. Immigration, HIV infection, and antiretroviral therapy in Italy. An epidemiological and clinical survey. Infez Med 2004; 12: 219-26

24. Beltrami C, Manfredi R, D'Antuono A, et al. Sexually-transmitted infections in adolescents and young adults in a large city of Northern Italy: a nine-year prospective survey. New Microbiol 2003; 26: $233-41$

25. Rockstroh JK, Gatell J, Landman R, et al. Management of late-presenting patients with HIV infection. Antivir Ther 2010; 15 (Suppl. 1): 25-30

26. Granich R, Crowley S, Vitoria M, et al. Highly active antiretroviral treatment as prevention of HIV transmission: review of scientific evidence and update. Curr Opin HIV AIDS 2010; 5: 298-304

27. Brent RJ, Brennan M, Karpiak SE. Economic evaluations of HIV prevention in rich countries and the need to focus on the aging of the HIV-positive population. Curr Opin HIV AIDS 2010; 5: 255-60

28. Verucchi G, Calza L, Manfredi R, et al. Incidence of liver toxicity in HIV-infected patients receiving isolated dual nucleoside analogue antiretroviral therapy. J Acquir Immune Defic Syndr 2003; 33: 546-8

29. Manfredi R. Management of dyslipidemia in patients with HIV disease. Clin Microbiol Infect 2000; 6: $579-84$

30. Manfredi R. High-risk dysmetabolism disorders associated with HAART-treated HIV disease, and reimbursement of lipid-lowering drugs, in a clinical and socio-economic perspective. AIDS Rev 2005; 7: 155-60

31. Manfredi R. Improvement of dyslipidemia during different HAART regimens: tenofovir- versus stavudine-containing antiretroviral combinations. Clin Infect Dis 2006; 42: 1345-7

32. Calza L, Manfredi R, Chiodo F. Hyperlactataemia and lactic acidosis in HIV-infected patients receiving antiretroviral therapy. Clin Nutr 2005; 24: 5-15

33. Manfredi R, Motta R, Patrono D, et al. A prospective case-control survey of laboratory markers of skeletal muscle damage during HIV disease and antiretroviral therapy. AIDS 2002; 16: 1969-71

34. Manfredi R, Chiodo F. A case-control study of virological and immunological effects of highly active antiretroviral therapy in HIV-infected patients with advanced age. AIDS 2000; 14: 1475-7

35. Manfredi R. HIV disease and advanced age: an increasing therapeutic challenge. Drugs Aging 2002; 19: 647-69

36. Manfredi R. Impact of HIV infection and antiretroviral therapy in the older patient. Expert Rev Anti Infect Ther 2004; 2: 821-4 
37. Verhelst D, Monge M, Meynard JL, et al. Fanconi syndrome and renal failure induced by tenofovir: a first case report. Am J Kidney Dis 2002; 40: 1331-3

38. Murphy MD, O’Hearn M, Chou S. Fatal lactic acidosis and acute renal failure after addition of tenofovir to an antiretroviral regimen containing didanosine. Clin Infect Dis 2003; 36: 1082-5

39. Karras A, Lafaurie M, Furco A, et al. Tenofovir-related nephrotoxicity in human immunodeficiency virus-infected patients: three cases of renal failure, Fanconi syndrome, and nephrogenic diabetes insipidus. Clin Infect Dis 2003; 36: 1070-3

40. Goicoechea M, Lì S, Best B, et al. Greater tenofovir-associated renal function decline with protease inhibitor-based versus nonnucleoside reverse-transcriptase inhibitor-based therapy. J Infect Dis 2008; 197: 102-8

41. Buchacz K, Young B, Baker RK, et al. Renal function in patients receiving tenofovir with ritonavir/ lopinavir or ritonavir/atazanavir in the HIV Outpatient Study (HOPS) cohort. J Acquir Immune Defic Syndr 2006; 43: 626-8

42. Martin J, Volberding P. HIV and premature aging: a field still in its infancy. Ann Intern Med 2010; 153: 477-9

43. Stenvinkel P. Chronic kidney disease: a public health priority and harbinger of premature cardiovascular disease. J Intern Med 2010; 268: 456-67. doi: 10.1111/j.1365-2796.2010.02269.x

44. Harris M. Nephrotoxicity associated with antiretroviral therapy in HIV-infected patients. Expert Opin Drug Saf 2008; 7: 389-400

45. Rho M, Perazella MA. Nephrotoxicity associated with antiretroviral therapy in HIV-infected patients. Curr Drug Saf 2007; 2: 147-54

46. Calza L, Manfredi R, Colangeli V, et al. Substitution of nevirapine or efavirenz for protease inhiibitors versus lipid-lowering therapy for the management of dyslipidaemia. AIDS 2005; 19: 1051-8

47. Manfredi R, Calza L. Recent availability of two novel, fixed formulations of antiretroviral nucleoside analogues: a 12-month prospective, open-label survey of their practical use and therapeutic perspectives in antiretroviral-naive and -experienced patients. AIDS Patient Care STDS 2008; 22: 279-90

48. Anuurad E, Semrad A, Berglund L. Human immunodeficiency virus and highly active antiretroviral therapy-associated metabolic disorders and risk factors for cardiovascular disease. Metab Syndr Relat Disord 2009; 7: 401-19

49. Guaraldi G, Baraboutis IG. Evolving perpectives on HIV-associated lipodystrophy syndrome: moving from lipodystrophy to non-infectious HIV co-morbidities. J Antimicrob Chemother 2009; 64: 437-40

50. Manfredi R. Need to evaluate the cost effectiveness of early diagnosis and treatment of osteopenia and osteoporosis in the setting of HIV and highly active antiretroviral therapy. AIDS Patient Care STDS 2006; 20: 665-7

51. Calza L, Tampellini L, Farneti B, et al. Bone mass loss in patients with human immunodeficiency virus type 1 infection: Association with male sex and protease inhibitor therapy Infect Dis Clin Pract 2007; 15: 160-6

52. Manfredi R. Polyunsaturated ethyl esters on n-3 fatty acids in HIV-infected patients with moderate hypertriglyceridemia: comparison with dietary and lifestyle changes, and fibrate therapy. $J$ Acquir Immune Defic Syndr 2004; 36: 878-80

53. Calza L, Colangeli V, Manfredi R, et al. Rosuvastatin for the treatment of hyperlipidaemia in HIVinfected patients receiving protease inhibitors: a pilot study. AIDS 2005; 19: 1103-5

54. Manfredi R, Calza L, Chiodo F. Hyperlactacidemia during antiretroviral therapy: frequency and clinical-therapeutical correlations. Infez Med 2006; 14: 33-6

55. Calza L, Manfredi R, Colangeli V, et al. Rosuvastatin, pravastatin, and atorvastatin for the treatment of hypercholesterolaemia in HIV-infected patients receiving protease inhibitors. Curr HIV Res 2008; 6: 572-8 
56. Manfredi R. Management of hypertrygliceridemia caused by combination antiretroviral therapy in HIV-infected patients: role of omega-3 polyunsaturated fatty acids at different dosages, compared with fibrates. Int J STD AIDS 2010; 21: 73-4

57. Jones R, Stebbing J, Nelson M, et al. Renal dysfunction with tenofovir disoproxil fumarate-containing highly active antiretroviral therapy regimens is not observed more frequently: a cohort and case-control study. J Acquir Immune Defic Syndr 2004; 37: 1489-95

58. Padilla S, Gutiérrez F, Masiá M, et al. Low frequency of renal function impairment during oneyear of therapy with tenofovir-containing regimens in the real-world: a case-control study. AIDS Patient Care STDs 2005; 19: 421-4

59. Zimmermann AE, Pizzoferrato T, Bedford J, et al. Tenofovir-associated acute and chronic kidney disease: a case of multiple drug interactions. Clin Infect Dis 2006; 42: 283-90

60. Marcotte S, Talbot A, Trottier B. Acute renal failure in four HIV-infected patients: potential association with tenofovir and nonsteroidal anti-inflammatory drugs. Can J Infect Dis Med Microbiol 2008; 19: 75-6

61. Mocroft A, Reiss P, Gasiorowski J, et al. Serious fatal and nonfatal non-AIDS-defining illnesses in Europe. J Acquir Immune Defic Syndr 2010; 55: 262-70

62. Izzedine H, Harris M, Perazella MA. The nephrotoxic effects of HAART. Nat Rev Nephrol 2009; 5: 563-73.

63. Post FA, Holt SG. Recent developments in HIV and the kidney. Curr Opin Infect Dis 2009; 22: 43-8

64. Borghi C, Dormi A, D’Addato S, et al. Brisighella Heart Study Working Party. Trends in blood pressure control and antihypertensive treatment in clinical practice: the Brisighella Heart Study. $J$ Hypertens 2004; 22: 1707-16

65. Scholze J, Alegria E, Ferri C, et al. Epidemiological and economic burden of metabolic syndrome and its consequence in patients with hypertension in Germany, Spain and Italy; a prevalencebased model. BMC Public Health 2010; 10: 529

66. Calza L, Manfredi R, Verucchi G. Myocardial infarction risk in HIV-infected patients: epidemiology, pathogenesis, and clinical management. AIDS 2010; 24: 789-802

67. Gruber VA, McCance-Katz EF. Methadone, buprenorphine, and street drug interactions with antiretroviral medications. Curr HIV/AIDS Rep 2010; 7: 152-60

68. Manfredi R, Sabbatani S, Agostini D. Trend of mortality observed in a cohort of drug addicts of the metropolitan area of Bologna, Nort-Eastern Italy, during a 25-year-period. Coll Antropol 2006; 30: $479-88$

69. Wyatt CM, Malvestutto C, Coca SG, et al. The impact of hepatitis C virus coinfection on HIVrelated kidney disease: a systematic review and meta-analysis. AIDS 2008; 22: 1799-807

70. Liss G, Rattan S, Lewis JH. Predicting and preventing acute drug-induced liver injury: what's new in 2010? Expert Opin Drug Metab Toxicol 2010; 6: 1047-61

71. Gorowara M, Burger D, Hill A, et al. Pharmacokinetics of low-dose protease inhibitors and efavirenz in low- and middle-income countries. Curr Opin HIV AIDS 2010; 5: 90-6

72. Calza L, Rosseti N, Biagetti C, et al. Abacavir-induced reaction with fever and severe skin rash in a patient tested human leukocyte-antigen-B ${ }^{\star} 5701$ negative. AIDS Patient Care STDS 2009; 23: 691-7

73. Kauf TL, Farkouh RA, Earnshaw SR, et al. Economic efficiency of genetic screening to inform the use of abacavir sulphate in the treatment of HIV. Pharmacoeconomics 2010; 28: 1025-39. doi: 10.2165/11535540-000000000-00000

74. Kiser JJ, Aquilante CL, Anderson PL, et al. Clinical and genetic determinants of intracellular tenofovir diphosphate concentrations in HIV-infected patients. J Acquir Immune Defic Syndr 2008; 47: 298-303

75. Best BM, Capparelli EV. Implications of gender and pregnancy for antiretroviral drug dosing. Curr Opin HIV AIDS 2008; 3: 277-82 
76. Post FA, Wyatt CM, Mocroft A. Biomarkers of impaired renal function. Curr Opin HIV AIDS 2010; 5: 524-30

77. Cruz DN, Bagshaw SM, Ronco C, et al. Acute kidney injury: classification and staging. Contrib Nephrol 2010; 164: 24-32

78. Sriwasat N, Hoste EE, Kellum JA. Modern classification of acute kidney injury. Blood Purif 2010; 29: $300-7$

79. [No author listed]. Emtricitabine/tenofovir disoproxil fumarate. Drugs R D 2004; 5: 160-1

80. Jenh AM, Thio CL, Pham PA. Tenofovir for the treatment of hepatitis B virus. Pharmacotherapy 2009; 29: 1212-27

81. Wiegand J, Van Bömmel F, Berg T. Management of chronic hepatitis B: status and challenges beyond treatment guidelines. Semin Liver Dis 2010; 30: 361-77

82. Thompson MA, Aberg JA, Cahn P, et al. Antiretroviral treatment of adult HIV infection: 2010 recommendations of the International AIDS Society-USA panel. JAMA 2010; 304: 321-33. doi: 10.1001/jama.2010.1004

83. Manfredi R, Sabbatani S, Fraternali Orcioni GF, et al. Fatal long-term immunosoppressive therapy with uncontrolled repeat prescription. Ther Drug Monit 2006; 28: 463-7

84. Castellano C, Williams W, Kepler TB, et al. Clinical predictors of tenofovir-associated nephrotoxicity in HIV-1-infected patients. XVII International AIDS Conference (AIDS 2008), Mexico City, 2008, abstract WEAB0104

85. Peyrière H, Reynes J, Rouanet I, et al. Renal tubular dysfunction associated with tenofovir therapy: report of 7 cases. J Acquir Immune Defic Syndr 2004; 35: 269-73

86. Créput C, Gonzalez-Canali G, Hill G, et al. Renal lesions in HIV-1-positive patient treated with tenofovir. AIDS 2003; 17: 935-7

87. Post FA, Moyle GJ, Stellbrink HJ, et al. Randomized comparison of renal effects, efficacy, and safety with once-daily abacavir/lamivudine versus tenofovir/emtricitabine, administered with efavirenz, in antiretroviral-naive, HIV-1-infected adults: 48-week results from the ASSERT study. J Acquir Immune Defic Syndr 2010; 55: 49-57

88. Goicoechea M, Liu S, Best B, et al. California Collaborative Treatment Group 578 Team. Greater tenofovir-associated renal function decline with protease inhibitor-based versus nonnucleoside reverse-transcriptase inhibitor-based therapy. J Infect Dis 2008; 197: 102

89. Perronne C. Antiviral hepatitis and antiretroviral drug interactions. J Hepatol 2006; 44(Suppl. 1): S119-S125

90. Verucchi G, Calza L, Manfredi R, et al. Human immunodeficiency virus and hepatitis C virus coinfection: epidemiology, natural history, therapeutic options and clinical management. Infection 2004; 32: 33-46

91. Verucchi G, Calza L, Biagetti C, et al. Ultrastructural liver mitochondrial abnormalities in HIV/ HCV-coinfected patients receiving antiretroviral therapy. J Acquir Immune Defic Syndr 2004; 35: 326-8

92. Calza L, Mosca L, Pocaterra D, et al. Assessing the impact of hepatitis C virus coinfection on lopinavi/ritonavir through concentrations in HIV-infected patients. Eur J CLin Pharmacol 2011; 67: 143-9

93. Pannu N, Nadim MK. An overview of drug-induced acute kidney injury. Crit Care Med 2008; 36 (Suppl. 4): S216-S223

94. Naggie S, Hicks C. Protease inhibitor-based antiretroviral therapy in treatment-naïve HIV-1-infected patients: the evidence behind the options. J Antimicrob Chemother 2010; 65: 1094-9

95. Bruggeman LA, Bark C, Kalavjian RC. HIV and the kidney. Curr Infect Dis Rep 2009; 11: 479-85

96. Manfredi R, Sabbatani S, Calza L. Antiretroviral therapy voluntary taken at half-dosage, but fully effective after 6-10 years: a provocative issue for adherence requirements. Curr HIV Res 2008; 6: 171-2 
97. Manfredi R, Calza L, Chiodo F. Dual nucleoside analogue treatment in the era of highly active antiretroviral therapy (HAART): a single-centre cross-sectional survey. J Antimicrob Chemother 2001; 48: 299-302

98. Manfredi R, Sabbatani S, Chiodo F. Long-term "self-managed" immunosuppressive treatment resulting in death due to fulminant hepatitis B: malpractice or patient's autolesionism? Clin Drug Invest 2005; 25: 615-20

99. Calza L, Manfredi R, Marinacci G, et al. Efficacy of penicillin G benzathine as antimicrobial treatment of cutaneous secondary syphilis in patients with HIV infection. J Chemother 2002; 14: 533-4

100. Sabbatani S, Manfredi R, Attard L, et al. Secondary syphilis presenting with severe hepatic involvement in a patient with undiagnosed HIV disease. AIDS Patient Care STDs 2005; 19: 545-9

101. Manfredi R, Sabbatani S, Pocaterra D, et al. Syphilis does not seem to involve virological and immunological course of concurrent HIV disease. AIDS 2006; 20: 305-6

102. Manfredi R, Calza L, Chiodo F. Changing epidemiology of hepatitis A in the Bologna metropolitan area, northern Italy: importance of counselling and prophylactic measures for the male homo/ bisexual population. Clin Microbiol Infect 2005; 11: 845-8

103. Verucchi G, Calza L, Attard L, et al. Acute hepatitis induced by traditional herbs used in the treatment of psoriasis. J Gastroenterol Hepatol 2002; 17: 1342-3

104. Kalyesubula R, Perazella MA. Nephrotoxicity of HAART. AIDS Res Treat 2011; 2011: 562790

105. Van Wiik JP, de Koning EJ, Cabezas MC, et al. Comparison of rosiglitazone and metformin for treating HIV lipodystrophy: a randomized trial. Arch Intern Med 2005; 143: 337-46

106. Raboud JM, Diong C, Carr A, et al. A meta-analysis of six placebo-controlled trials of thiazolidinedione therapy for HIV lipoatrophy. HIV Clin Trials 2010; 11:39-50

107. Sheth SH, Larson RJ. The efficacy and safety of insulin-sensitizing drugs in HIV-associated lipodystrophy syndrome: a meta-analysis of randomized trials. BMC Infect Dis 2010; 10: 183

108. Libório AB, Andrade L, Pereira LV, et al. Rosiglitazone reverses tenofovir-induced nephrotoxicity. Kidney Int 2008; 74: 910-8

109. Calza L. Long-term use of rosuvastatin: a critical risk benefit appraisal and comparison with other antihyperlipidemics. Drug Healthc Patient Saf 2009; 1: 25-33

110. Audia P, Feinfeld DA, Dubrow A, et al. Metformin-induced lactic acidosis and acute pancreatitis precipitated by diuretic, celecoxib, and candersartan acute kidney dysfunction. Clin Toxicol (Phila) 2008; 46: 164-6

111. Spiller HA, Sawyer TS. Toxicology of oral antidiabetic medications. Am J Health Syst Pharm 2006; 63: 929-38

112. Devetzis V, Passadakis P, Panagoutsos S, et al. Metformin-related lactic acidosis in patients with acute kidney injury. Int Urol Nephrol 2011; 43: 1243-8

113. Agrawal V, Shah A, Rice C, et al. Impact of treating the metabolic syndrome on chronic kidney disease. Nat Rev Nephrol 2009; 5: 520-8

114. Petric M, Tasic L, Sukljevic S. Nonsteroideal anti-inflammatory drug usage and gastrointestinal outcomes in the Republic of Serbia. J Pain Palliat Care Pharmacother 2009; 23: 40-7

115. Wood DM, Monaghan J, Streete P, et al. Fatality after deliberate ingestion of sustained-release ibuprofen: a case report. Crit Care 2006; 10: R44

116. Lobitz S, Velleuer E. Guido Fanconi (1892-1979): a jack of all trades. Nat Rev Cancer 2006; 6: 893-8

117. Blattner WA, Biggar RJ, Weiss SH, et al. Epidemiology of human T-lymphotropic virus type III and the risk of the acquired immunodeficiency syndrome. Ann Intern Med 1985; 103: 665-70

118. El-Sadr WM, Lundgren JD, Neaton JD, et al. CD4+ count-guided interruption of antiretroviral treatment. N Engl J Med 2006; 355: 2283-96 
119. Phillips AN, Carr A, Neuhaus J, et al. Interruption of antiretroviral therapy and risk of cardiovascular disease in persons with HIV-1 infection: exploratory analyses from the SMART trial. Antivir Ther 2008; 13: 177-87

120. Worm SW, Sabin C, Weber R, et al. Risk of myocardial infarction in patients with HIV infection exposed to specific individual antiretroviral drugs from the 3 major drug classes: the data collection on adverse events of anti-HIV drugs (D: A: D) study. Antivir Ther 2008; 13: 177-87

121. Manfredi R. Multicentre inter-cohort studies - reliable responses for HIV disease management? AIDS Rev 2009; 11: 53-5

122. Manfredi R. Hyperinflation of quoted co-Authors in observational and clinical studies, intercohort and pooled analyses, in the field of HIV disease. J Acquir Immune Defic Syndr 2011; 56: e56-8

123. Caron-Debarle M, Lagathu C, Boccara F, et al. HIV-associated lipodystrophy: from fat injury to premature aging. Trends Mol Med 2010; 16: 218-29

124. Levey AS, Bosch JP, Lewis JB, et al. A more accurate method to estimate glomerular filtration rate from serum creatinine: a new prediction equation. Ann Intern Med 1999; 130: 461-70

125. Levey AS, Steven LA, Schmid CH, et al. A new equation to estimate glomerular filtration rate. Ann Intern Med 2009; 150: 604-12

126. John R, Herzenberg AM. Renal toxicity of therapeutic drugs. J Clin Pathol 2009; 62: 505-15

127. Venkatachalam MA, Griffin KA, Lan R, et al. Acute kidney injury: a springboard for progression in chronic kidney disease. Am J Physiol Renal Physiol 2010 (March 3) [Epub ahead of print]

128. Mori T, Cowley AW Jr, Ito S. Molecular mechanisms and therapeutic strategies of chronic renal injury: physiological role of angiotensin II-induced oxidative stress in renal medulla. J Pharmacol Sci 2006; 100: 2-8

129. Spiller HA, Sawyer TS. Toxicology of oral antidiabetic medications. Am J Health Syst Pharm 2006; 63: 929-38

130. Perrone J, Phillips C, Gaieski D. Occult metformin toxicity in three patients with profound lactic acidosis. J Emerg Med 2011; 40: 271-5

131. Goicoechea M, Jain S, Bi L, et al. Abacavir and tenofovir disoproxil fumarate co-administration results in a nonadditive antiviral effect in HIV-1-infected patients. AIDS 2010; 24: 707-16

132. Centers for Disease Control and Prevention (CDC). Twenty-five years of HIV/AIDS-United States, 1981-2006. MMWR 2006; 55: 585-9

133. Waters LJ, Nelson MR. Are all non-thymidine analogue backbones appropriate for treating antiretroviral-naïve patients? Int J Clin Pract 2005; 59: 1452-8

134. Stürmer M, Staszewski S, Doerr HW. Quadruple nucleoside therapy with zidovudine, lamivudine, abacavir and tenofovir in the treatment of HIV. Antivir Ther 2007; 12: 695-703

135. Schafer JJ, Squires KE. Integrase inhibitors: a novel class of antiretroviral agents. Ann Pharmacother 2010; 145-56

136. Lefèvre C, Auclair M, Boccara F, et al. Premature senescence of vascular cells is induced by HIV protease inhibitors. Implication of prelamin A and reversion by statin. Arterioscler Thromb Vasc Biol 2010; 30: 2611-20. doi: 10.1161/ATVBAHA.110.213603

137. Manfredi R, Chiodo F. Substitution of protease inhibitors during treatment of patients with human immunodeficiency virus infection: frequency, mode, reasons, and mid-term outcome. J Antimicrob Chemother 2000; 45: 261-3

138. Manfredi R, Chiodo F. Switch of protease inhibitor-containing HAART in routine clinical practice: a four-year prospective observational study. Int J STD AIDS 2001; 12: 84-8

139. Sabbatani S, Manfredi R, Fulgaro C, et al. Reversal of major genotypic tipranavir mutations under long-term treatment with tipranavir/ritonavir itself with very limited optimized background, during deep salvage antiretroviral therapy. Curr HIV Res 2009; 311-3 
140. Palacios R, Hidalgo C, Ríos MJ, et al. Effectiveness and safety of simplification from tenofovirlamivudine (TDF-3TC) to tenofovir-emtricitabine (TDF-FTC) co-formulation (Truvada) in virologically suppressed HIV-infected patients on HAART. Eur J Clin Microbiol Infect Dis 2009; 28: 399-402

141. Simpson KN. Economic modeling of HIV treatments. Curr Opin HIV AIDS 2010; 5: 242-8

142. Kearney BP, Yale K, Shah J, et al. Pharmacokinetics and dosing recommendations of tenofovir disoproxil fumarate in hepatic or renal impairment. Clin Pharmacokinet 2006; 45: 1115-24

143. Esté JA, Cihlar T. Current status and challenges of antiretroviral research and therapy. Antiviral Res 2010; 85: 25-33. http://dx.doi.org/10.1016/j.antiviral.2009.10.007

144. Palumbo PE, Kwok S, Waters S, et al. Viral measurement by polymerase chain reaction-based assays in human immunodeficiency virus-infected infants. J Pediatr 1995; 26: 592-5

145. Vella S. Clinical experience with saquinavir. AIDS 1995; 9(Suppl 2): S21- S25 